NBER WORKING PAPER SERIES

\title{
PRICE INDEXES FOR CLINICAL TRIAL RESEARCH: A FEASIBILITY STUDY
}

\author{
Ernst R. Berndt \\ Iain M. Cockburn \\ Working Paper 18918 \\ http://www.nber.org/papers/w18918
NATIONAL BUREAU OF ECONOMIC RESEARCH
1050 Massachusetts Avenue
Cambridge, MA 02138
March 2013

Research support from the National Science Foundation, Collaborative Research: "An Experimental Producer Price Index for Clinical Trials" to the National Bureau of Economic Research, Award Number 0915674, and Boston University, Award Number 0915677, is gratefully acknowledged. We thank Medidata Solutions Worldwide, Inc. ("Medidata") for supplying database extracts, Ed Seguine (formerly at Fast Track Solutions, Inc. and Medidata) for helpful discussions, Rafael Campo and other individuals at Medidata for helpful comments and data support, and Marshall Reinsdorf from the Bureau of Economic Analysis for constructive discussant's comments at the Annual Meetings of the American Economic Association in San Diego, CA, January 6, 2013. Rania Gihleb provided able research assistance. The opinions expressed herein are those of the authors, and do not necessarily reflect those of the National Science Foundation, Medidata, or the institutions with whom the authors are affiliated. The views expressed herein are those of the authors and do not necessarily reflect the views of the National Bureau of Economic Research.

NBER working papers are circulated for discussion and comment purposes. They have not been peerreviewed or been subject to the review by the NBER Board of Directors that accompanies official NBER publications.

(C) 2013 by Ernst R. Berndt and Iain M. Cockburn. All rights reserved. Short sections of text, not to exceed two paragraphs, may be quoted without explicit permission provided that full credit, including (C) notice, is given to the source. 
Price Indexes for Clinical Trial Research: A Feasibility Study

Ernst R. Berndt and Iain M. Cockburn

NBER Working Paper No. 18918

March 2013, Revised September 2013

JEL No. E31,L65,O49

\begin{abstract}
$\underline{\text { ABSTRACT }}$
We estimate hedonic price indexes for clinical trial research, an important component of biomedical R\&D, using a large sample of agreements between trial sponsors and clinical investigators obtained from Medidata Solutions Worldwide Inc. Nominal prices measured as total grant cost per patient rose by a factor of 4.5 between 1989 and 2011, while the NIH Biomedical R\&D Price Index (BRDPI) focused on input costs rose only 2.2-fold. Most of the disparity appears to be attributable to changes in the nature and organization of clinical trials: during this period the average number of patients per site fell substantially while "site work effort" more than doubled. After controlling for these changes in the characteristics of investigator agreements using a variety of methods based on hedonic regressions, we find that the estimated rate of inflation in clinical trials costs tracks the BDRPI very closely. Results from this study suggest that it should be feasible for statistical agencies to develop a producer price index for this type of R\&D activity, contributing to broader efforts to develop a deflator for contracted R\&D services.
\end{abstract}

Ernst R. Berndt

MIT Sloan School of Management

100 Main Street, E62-518

Cambridge, MA 02142

and NBER

eberndt@mit.edu

Iain M. Cockburn

School of Management

Boston University

595 Commonwealth Ave

Boston, MA 02215

and NBER

cockburn@bu.edu 


\section{Introduction}

R\&D expenditures are widely acknowledged to a play a key role in economic growth and competitiveness, and statistics on $\mathrm{R} \& \mathrm{D}$ are closely watched as indicators of technological change and national economic performance. ${ }^{1}$ Yet R\&D has historically been treated in the national accounts as a business expense, rather than as an investment in knowledge capital, which has important implications for estimates of GDP and GDP growth. As a first step in treating R\&D as an investment component of GDP, the Bureau of Economic Analysis has created an R\&D satellite account, which reports that $\mathrm{R} \& \mathrm{D}$ contributed 20 basis points to the re-estimated $2.9 \%$ average rate of real GDP growth from 1957 to 2007. ${ }^{2}$ But such calculations can be quite sensitive to the use of deflators, and the nature of $R \& D$ activities present substantial price measurement challenges to statistical agencies. Although BLS has steadily expanded the scope of its collection of price statistics for the service sector, it does not currently publish a PPI for business R\&D services, a major component of total R\&D activity in the US. ${ }^{3}$ In experimenting with the satellite $R \& D$ accounts, the BEA has utilized various proxy deflators to construct measures of real business R\&D output, including cost-based aggregate indexes for inputs to $R \& D$ (that implicitly assume no productivity growth in $R \& D$ activities), weighted combinations of gross output prices of industries investing in $\mathrm{R} \& \mathrm{D}$, and a variety of other next best alternatives in lieu of actual R\&D output prices. ${ }^{4}$ Were a broad-based PPI for contracted business R\&D available, this could be used to develop estimates of real private sector R\&D output by deflating

\footnotetext{
${ }^{1}$ Although the United States has long led the world in combined government and industry spending on research and development $(R \& D)$, in recent years Asian economies have increased their R\&D to GDP intensity, resulting in $R \& D$ levels that have gained considerable ground on that of the U.S. One estimate has 2012 US R\&D expenditures of $\$ 418.6$ billion and an R\&D/GDP ratio of $2.68 \%$, while comparable numbers for China are \$197.3 billion and 1.60\%, and for Japan \$159.9 billion and 3.48\% Other countries with 2012 R\&D/GDP ratios of greater than 3\% include Finland at 3.80\%, Sweden 3.62\%, South Korea 3.45\% and Denmark 3.08\%. Source: Battelle Institute, R\&D Magazine, 2012 Global R\&D Funding Forecast "2012 Global R\&D Funding Forecast", p. 5, December 2011. Available online at http://www.battelle.org/docs/default-document-library/2012 global forecast.pdf, last accessed 11 January 2013.

${ }^{2}$ Lee, J and Schmidt, AG. "Research and Development Satellite Account Update: Estimates for 1959-2007" Survey of Current Business, 90(12):16-27. December 2010

${ }^{3}$ Industry performs about $71 \%$ of R\&D in the US. Battelle Institute, R\&D Magazine, 2012 Global R\&D Funding Forecast, p. 6.

${ }^{4}$ David Friedman [2010], "Developing a PPI for Scientific Research and Development NAICS 5417/ISIC 7310", presentation at the $25^{\text {th }}$ Meeting of the Voorburg Group, Vienna, Austria, 23 September 2010. Available online at http://stds.statcan.ca/english/voorburg/Documents/2010\%20Vienna/Papers/2010\%20-\%2081.pdf, last accessed 13 January 2013, slide 6; also see Adam Copeland and Dennis Fixler, "Measuring the Price of Research and Development Output", paper presented at the National Bureau of Economic Research, Summer Institute Productivity Program, J:uly 2008; and Adam Copeland, Gabriel W. Medeiros and Carol A. Robbins, "Estimating Prices for R\&D Investment in the 2007 R\&D Satellite Accounte Background Paper, November 2007, both available at www.bea.gov.
} 
a portion of the Census Bureau's NAICS 5417 nominal expenditure estimates for "Scientific Research and Development Services”. As noted by BLS Assistant Commissioner David Friedman, however, what is desired is an ideal PPI "that directly measures actual market transactions for R\&D output". 5

This paper contributes to this broader effort by studying measurement of such R\&D transactions prices for the specific area of biomedical clinical research. Biomedical research is the single biggest component of the R\&D sector's estimated contribution to growth: in the BEA's initial estimates in the satellite account, biotechnology related industries contributed 44\%,of the total business R\&D contribution to real GDP growth between 1998 and 2007, with information-communications-technology producing industries contributing $36 \%$, transportation equipment industries $11 \%$ and all other industries $9 \%{ }^{6}$ Within biomedical research, clinical trials account for a large fraction of commercial $R \& D$ expenditure in this sector: of the $\$ 46.4$ billion spent by Pharmaceutical Research and Manufacturers of America (PhRMA) member companies in 2010 on R\&D, $\$ 32.5$ billion (70\%) was spent on clinical trials involving human subjects, with a much smaller proportion devoted to pre-clinical and basic research. ${ }^{7}$ In recent years, clinical research has accounted for about $1 / 3$ of the total NIH budget ( $\$ 10.7$ billion out of $\$ 30$ billion in FY2010) of which a substantial fraction ( $\$ 3.2$ billion in FY2010) is expenditure on clinical trials. ${ }^{8}$

Clinical research is also an activity which has seen significant organizational, technological and economic changes, with potentially important implications for productivity. Over the past three decades, the design and management of clinical trials has become increasingly sophisticated, and at the same time the types of organizations conducting clinical trials have changed. Much of the effort in running clinical trials is increasingly contracted out to specialist entities called contract research organizations (CROs) rather than being incurred "in house" or by biopharmaceutical companies in collaborations with external academic medical center investigators. Moreover, within the US, sponsors are moving trials away from academic

\footnotetext{
${ }^{5}$ Friedman [2010], slide 6.

${ }^{6}$ Friedman [2010], Slide 5.,

${ }^{7}$ Pharmaceutical Research and Manufacturers of America, Pharmaceutical Industry Profile 2011, Washington DC, PhRMA, April 2011, Appendix Table 5, p. 45. Expenditure on Phase 1 trials was \$3.753 billion, Phase 2 \$7.124 billion, Phase $3 \$ 16.300$ billion, and Phase 45.303 billion.

${ }^{8}$ See http://report.nih.gov/rcdc/categories/default.aspx, visited 11/30/2011.
} 
medical centers toward physician practices, many of them for-profit. ${ }^{9}$ For example, while the number of clinical research contracts carried out annually by academic investigators remained relatively constant at about 3,000 between 1991 and 1999, over the same time period the number of trial research contracts with non-academic investigators increased from about 1,700 to 5,000.

Importantly, multi-site clinical trials have also become increasingly global in nature, recruiting patients in many countries simultaneously. For a large Phase III trial evaluating cardiovascular or central nervous system drugs, more than 10,000 patients will typically be recruited at 100-200 sites in ten or more countries worldwide. ${ }^{10}$ The size of dossiers filed by biopharmaceutical firms in support of New Drug Applications (NDAs) at the US Food and Drug Administration has increased over time, reflecting increased complexity and detailed sitespecific information: the mean number of pages per NDA increased from 38,000 in 1977-80 to 56,000 in $185-88$ and 91,000 in 1989-92. ${ }^{11}$

To date, very little information has been available on trends in the pricing of the R\&D services that are the inputs to clinical trials. In 1985 the U.S. Bureau of Labor Statistics began publishing producer price indexes (PPIs) for various service sector industries, an effort which has expanded to include including PPIs for aspects of health care delivery such as hospitals and physician services $^{12}$. However these indexes do not cover contracted business R\&D. To date, the only published source of information on trends in pricing in the biomedical R\&D sector is the Biomedical R\&D Price Index (BRDPI) constructed by the BEA under contract with the National Institutes of Health (NIH). The BRDPI is based on a chained Laspeyres methodology using micro budget data from individual NIH investigator grants. This index measures changes in the weighted-average of the prices of all the inputs (e.g., personnel services, various supplies, and equipment) that are purchased or leased with the NIH budget to support research. Input weights

\footnotetext{
${ }^{9}$ See Pierre Azoulay, "Capturing Knowledge Within and Across Firm Boundaries: Evidence from Clinical Development”, American Economic Review 94(5):1591-1612, December 2004. Available at

http://pazoulay.scripts.mit.edu/pubs/knowledge.pdf; Pierre Azoulay and Ariel Fishman, "Doctors, \$\$ and Drug Development: The Rise for For-Profit Experimental Medicine", paper presented at the National Bureau of Economic Research Location of Biopharmaceutical Activity Conference, Savannah, GA, March 7-8, 2008. Available from pazoulay@mit.edu

${ }^{10}$ See, for example, Fabio Thiers, Anthony J. Sinskey and Ernst R. Berndt, "Trends in the Globalization of Clinical Trials", Nature Reviews: Drug Discovery 7(1):13-14, 2008. doi:10-1038/nrd2441.

${ }^{11}$ Pierre Azoulay, "The Changing Economics of Clinical Development”, presentation to the Earth Institute, Columbia University, May 20, 2004, slide 7. Available from pazoulay@ mit.edu.

${ }^{12}$ Roslyn Swick, Deanna Bathgate and Michael Horrigan, "Services Producer Price Indices: Past, Present, and Future", paper presented at the Federal Economic Statistics Advisory Committee on June 9, 2006.
} 
reflect the changing actual shares of total NIH expenditures on each of the types of inputs purchased. ${ }^{13}$ According to the NIH,

"Theoretically, the annual change in the BRDPI indicates how much NIH expenditures would need to increase, without regard to efficiency gains or changes in government priorities, to compensate for the average increase in prices due to inflation and to maintain NIH-funded research activity at the previous year's level.",14

The BRDPI is published annually on a federal government fiscal year (October 1 - September 30) basis and reaches back to 1950.

In addition to contributing to the broader effort to construct measures of real R\&D output, the development of a quality-constant PPI for clinical R\&D would provide insights on important policy issues specific to this sector. For example, while total R\&D spending by PhRMA member companies has almost doubled over the last decade ${ }^{15}$ (as has the overall NIH budget $^{16}$ ), the number of new drugs and biologics approved by the FDA each year in the last decade has not yet returned to its mid-1990s peak levels. One prominent study reports that the capitalized cost of bringing a new drug to market, adjusted for general inflation in year 2000 dollars, more than doubled from \$318 to \$802 million between 1991 and 2003. ${ }^{17}$

This raises some very basic — and as yet unanswered — questions. Increases in the cost per approved drug are often equated with "the price of innovation", but in fact little is known about how much of the increase in expenditure reflects changes in the prices of inputs to biomedical research and how much reflects changes in the quantity and complexity of research being performed. Have the prices of inputs to clinical research increased more rapidly than overall inflation, or are these inputs being used more intensively, or are both occurring? Moreover, to what extent has the "quality" of inputs changed? The growing complexity of

\footnotetext{
${ }^{13}$ For details on the construction of the BRDPI, see James Schuttinga, "The Biomedical Research and Development Price Index", powerpoint presentation for MIT economists, March 25, 2008; National Institutes of Health, Office of Budget, "Biomedical Research and Development Price Index (BRDPI): Fiscal Year 2010 Update and Projections for FY 20110FY2016, Press Release, January 24, 2011. Available online at http://officeofbudget.od.nih.gov/pdfs/FY12/BRDPI_Proj_Jan_2011_Final.pdf..

${ }^{14}$ National Institutes of Health [2011], p. 1.

${ }^{15}$ Total R\&D spending by PhRMA member companies was $\$ 26.0$ billion in 2000, and $\$ 49.4$ billion in 2010, an increase of 90\%. Pharmaceutical Research and Manufacturers of America [2011], Appendix Table 1, p. 42.

${ }^{16}$ The total NIH budget obligations in fiscal year 2000 was $\$ 17.8$ billion, and $\$ 31.0$ billion in fiscal year 2010, an increase of 74\%. National Institutes of Health, Mechanism Detail, Actual Obligations, available at http://officeofbudget.od.nih.

$\frac{17}{17}$ Joseph A. DiMasi, Ronald W. Hansen and Henry G. Grabowski, "The Price of Innovation: New Estimates of Drug Development Costs”, Journal of Health Economics 22(2):151-85, March 2003.
} 
clinical trials and the underlying science suggests that more time, more highly trained personnel, and more sophisticated equipment may be required to conduct a typical study. ${ }^{18}$

Very little data is currently available to inform discussion of such issues. While data are captured for some inputs to clinical research, such as salaries of post-doctoral fellows, relatively little is known about other important inputs to clinical research such as site administration costs, computational time, materials and investigator salaries. Critically, even where good data are available on input prices, it is important to take into account how inputs are combined by focusing on an appropriate unit of analysis. ${ }^{19}$

More generally, in the language of the economics of price measurement, what is needed is measures of "constant-quality" changes in prices and quantities, i.e., holding the characteristics of the input and output activities constant when looking at changes in expenditures over time or cross-sectionally. Failure to do so can result in quite misleading interpretations and policy recommendations. Analyses of expenditures on personal computers, for example, recognize that there have been huge increases in the performance or capacity of the products sold, but very small changes in their nominal prices; "constant quality" prices have thus fallen substantially over time — various estimates suggest sustained real price declines of more than $25 \%$ per year over several decades. ${ }^{20}$ Various governmental statistical agencies now routinely take this phenomenon into account for many types of information technology and other electronic goods in developing estimates of GDP, with quite marked impacts on measures of economic growth and productivity. ${ }^{21}$

While it is important, therefore, to quantify the "price" versus "quantity" component changes in $\mathrm{R} \& \mathrm{D}$, adjusting both for quality, characterizing scientific research presents some very

\footnotetext{
${ }^{18}$ Kenneth A. Getz, Julia Wenger, Rafael Campo, Eward S. Seguine and Kenneth L. Kaitlin, "Assessing the Impact of Protocol Design Changes on Clinical Trial Performance", American Journal of Therapeutics 15(5):450-57, 2008.

${ }^{19}$ See, for example, the highly influential studies by Cutler and coauthors on the costs of treating heart attacks which has had a major impact on analyses of health expenditures by focusing attention on changes in the cost of an "episode of care" due to input substitution, rather than on changes in the per unit-price of inputs to care. See, for example, David Cutler [1998], “Are Medical Prices Falling?", Quarterly Journal of Economics, November 9911024; David Cutler and Mark McClellan [1998], "What Is Technological Change?", in David Wise, ed., Inquiries in the Economics of Aging, Chicago: University of Chicago Press for the NBER; and David Cutler and Mark McClellan [2001], "Is Technological Change in Medicine Worth It?", Health Affairs 20(1):11-29.

${ }^{20}$ See, for example, Ernst R. Berndt and Neal J. Rappaport, "Price and Quality of Desktop and Mobile Personal Computers: A Quarter Century Historical Overview", American Economic Review 91(2):268-73, May 2001, and the references cited therein.

${ }^{21}$ For more detailed discussion, see ch. 4 in Charles L. Schultze and Christopher Mackie, eds., At What Price? Conceptualizing and Measuring Cost-of-Living and Price Indexes, Washington DC: National Academy Press for the National "Academy of Sciences, 2002.
} 
substantial measurement problems. Research activities are typically highly heterogeneous and idiosyncratic in nature, drawing on quite different inputs and resources to produce "output" which is very difficult to measure consistently. However in one respect, clinical trials may be unusually tractable. Clinical development is a highly structured activity, in which individual "experiments" are relatively well-defined and activity is closely tracked. Industry trends are also creating an unusual opportunity to investigate these questions. While biopharmaceutical companies and non-profit entities continue to be the lead sponsors of clinical trials, much of the effort in conducting them is increasingly outsourced to contract research organizations ("CROs") rather than being incurred in-house. At least within the US, the investigators who recruit, treat, and observe subjects are drawn less from academic medical centers and increasingly more from independent physician practices. ${ }^{22}$ This has meant that data on contractual terms among all these parties are now ever more important and increasingly visible.

We report here results from a study directed at assessing the feasibility of constructing a PPI for clinical trial research, based on actual transactions data involving CRO contracts. Specifically, we analyse a sample of over 215,000 contracts regarding payments made by trial sponsors (directly or through CRO intermediaries) to clinical investigators and study sites from the PICAS ${ }^{\circledR}$ database maintained by Medidata Worldwide Solutions, Inc. This sample covers over 24,000 distinct Phase I through Phase IV clinical study protocols conducted between 1989 and 2011 in 52 different countries. Using information on the protocol characteristics, we estimate parameters in multiple regression equations and compute hedonic price indexes that allow us to estimate the rate of inflation in this particular aspect of clinical research, controlling for changes in the characteristics of clinical trials over the sample period. Because hedonic price indexes based on regression equations estimated using data pooled over time would entail revising the historical price index time series each time another time period of data was added to the sample, we also investigate use of chained indexes based on sequential "adjacent year" regressions, and Paasche, Laspeyres, and Fisher Ideal indexes based on single-year regression equations and from utilizing the "pure hedonics" approach in which year-on-year changes in the estimated price of characteristics are weighted by once-lagged (Laspeyres) or current period (Paasche) characteristics quantities. ${ }^{23}$

\footnotetext{
${ }^{22}$ Azoulay [2004], and Azoulay and Fishman [2008].

${ }^{23}$ We thank Marshall Reinsdorf, the discussant of a previous version of this paper, for this suggestion. The method we utilize was presented by Ariel Pakes and by Ernst Berndt, Zvi Griliches and Neal J. Rappaport. See Ariel Pakes,
} 
We find that while our measure of unit costs of this aspect of conducting clinical trials rose rapidly over the two decades covered by this sample at about 8\% per year (roughly twice the rate of inflation in the NIH's Biomedical R\&D Price Index), these changes in nominal costs appear to be driven by a variety of factors other than input costs. At least in this sample there has been a substantial increase in the level of effort required by investigators, and significant changes in both the composition of the sample across therapeutic classes and stages of clinical development, as well as in the organization of trials with a trend towards smaller numbers of patients per site and considerable variation over time in the geographic distribution of ex-US sites. After controlling for these factors using a variety of hedonic regression methods, we find much lower growth rates in costs, with adjusted rates of inflation between $1 / 3$ and $2 / 3$ lower than those seen in the unadjusted data. Interestingly, we find that growth of the price index based on our preferred hedonic price regression specification using US data between 1989 and 2011 is virtually identical to that of the BRDPI.

\section{Data}

With the co-operation of Medidata Worldwide Solutions, Inc. ("Medidata"), we assembled a dataset of 216,076 observations on "investigator grants," which are payments made by a trial sponsor to the individual investigators or "sites" that enroll subjects. ${ }^{24}$ These payments cover the investigators' costs of recruiting subjects, administering the treatments, measuring clinical endpoints, etc., plus overhead allowances reflecting payments for the use of the site's facilities. We focus on the total grant cost per patient ("TGPP") as the economically meaningful unit of analysis for understanding price trends. Total grant cost per patient is the total amount paid by the sponsor under its contract with the site, divided by the number of patients planned to be enrolled at that site. For about $12 \%$ of the records contained in the PICAS ${ }^{\circledR}$ database, the contract specifies only a per-patient amount, not the number of patients. These contracts are excluded from the results reported below (although we apply certain robustness checks), since

\footnotetext{
“A Reconsideration of Hedonic Price Indexes with an Application to PCs", American Economic Review 93(5):15781614, December 2003, and by Ernst R. Berndt, Zvi Griliches and Neal J. Rappaport, "Econometric Estimates of Price Indexes for Personal Computers in the 1990s", Journal of Econometrics 68(1):243-268, July 1995, and the references cited therein.

${ }^{24}$ The dataset was originally compiled by Fast Track Systems; Medidata acquired Fast Track in 2007. For further details see Fast Track Systems, Grants Manager Data Dictionary, Conshohocken, PA: Fast Track Systems, Inc. Excel spreadsheet file, 2006 and Medidata Solutions Worldwide, "Medidata Solutions Partners with Fast Track Systems to Accelerate Enterprise-Wide Deployment of Electronic Clinical Trials", press release, February 7, 2007-, accessed 7 December 2008.
} 
we are unable to control for the scale of the site's effort. For ex-US sites where the contract is in a foreign currency, we convert to US dollars using the spot exchange rate. Typically these payments make up about half of the total cost of a trial, the remainder being headquarters' "overhead" in the form of trial design, data management and analysis, site selection and monitoring, etc., by the sponsors.

Table I shows a summary of the number of records in the dataset by year each investigator contract was signed, along with summary statistics for the TGPP. The dataset used here covers the almost quarter century period 1989 to 2011. The number of records per year varies over time, with the period 1992-2002 accounting for almost $75 \%$ of the total number of records. The number of records per year in our sample reached a peak in 2000 and declines steadily thereafter, reflecting two factors. First, the PICAS® database was originally compiled from an archive of paper records, and has since transitioned to electronic source documents. This transition led to a temporary decline in the number of contributions from the participating organizations in 2004-2005. Second, the fraction of contracts that do not specify the number of patients expected to be enrolled at the site (and are excluded from our sample) has increased over the past decade. This trend likely reflects tighter "real time" tracking and control of patient enrollment by trial sponsors and challenges in sites being equally able to enroll planned number of patients at their sites.

As can be seen from the table, the mean TGPP rises quite rapidly in nominal terms, just over four-fold over the period 1989 to 2011, from $\$ 3773$ to $\$ 16567$, with an average annual growth rate (AAGR) of $7.5 \% ;^{25}$ the median value of each year increases slightly more rapidly, from $\$ 2779$ to $\$ 13222$, an AAGR of $8.2 \%{ }^{26}$ By comparison, between fiscal years 1989 and 2011 the NIH's BRDPI increased much more slowly, barely doubling at an AAGR only half as large at $3.7 \% .^{27}$ The distribution of TGPP is quite skewed, with the median somewhat below the mean value; a visual plot suggests that TGPP can be reasonably approximated with the lognormal distribution. Notably, the within-year coefficient of variation is relatively large but stable at around 0.80 at both the beginning and end of the sample period. While we attempt to account for this variation in TGPP with measured site and protocol characteristics, some part is

\footnotetext{
${ }^{25}$ These AAGRs are literally the arithmetic means of year-over-year growth rates; the compounded average annual growth rate (CAGR) is slightly less, at $7.0 \%$; see note 40 below.

${ }^{26}$ In the somewhat larger sample $(245,803$ records) that includes contracts where the number of patients is not specified, figures are very similar: mean TGPP rises from $\$ 3,752$ to $\$ 15,567$ at an AAGR of $7.1 \%$.

${ }^{27}$ National Institutes of Health [2011], Supplemental Table A, p. 6.
} 
likely attributable to factors such as the conversion of foreign transactions to US \$ using the spot exchange rate at the time of the transaction.

Table II and Figures 1 and 2 show two important aspects of trials that impact the costs incurred by an investigator: site work effort and number of patients. Site work effort (SWE) is a patent-pending measure of clinical trial complexity and burden developed by Medidata. SWE was constructed as follows. Based on examination of detailed protocols, inclusion and exclusion criteria, the number and use intensity of various procedures such as laboratory tests, blood work, questionnaires and subjective assessments, office consultations and examinations, and use of diagnostic technologies such as X-rays, imaging or heart activity assessments, relative value units $\left(\right.$ RVUs) ${ }^{28}$, or where unavailable or inapplicable, comparable Work Effort Unit (WEUs) created by Medidata in conjunction with researchers at the Tufts Center for Study of Drug Development, were assigned to each procedure in a trial protocol. A complexity measure was computed simply as the number of distinct procedures in the trial protocol. An aggregate investigative SWE measure was then computed as the cumulated product of the number and intensity in use of these procedures, in RVU/WEU units, conducted over the course of the entire protocol for each of the trials. ${ }^{29}$ It is important to note that SWE is therefore a protocol-level measure of the work effort required from each site, and that actual resources used by each site in implementing the protocol may differ to some degree. Table II panel (a) and Figure 1 report descriptive statistics for SWE for the 24,236 distinct protocols in our sample. As can be seen there, mean and median values of SWE have increased very significantly over time, with the mean value per protocol rising almost three-fold between 1989 and 2011, at AAGRs of 5.2\% (mean) and 6.1\% (median). ${ }^{30}$

By contrast, as seen in panel (b) of Table II and in Figure 2, the mean number of patients per site has fallen substantially over the same period from 25.48 to 11.83 , a factor of about two, whereas the median has fallen more dramatically, from 20 to eight. The relative volatility (coefficient of variation) of number patients per site has increased steadily, from about 1.3 in 1989 to about 1.7 in $1999-2000$ and to 2.0 in 2010, while that for SWE has been relatively stable at about 0.7 .

\footnotetext{
${ }^{28}$ RVUs are measures constructed by Medicare to estimate the relative level of physician time, skill, training, and expertise and required equipment, supplies, rent and office staffing costs for conducting procedures, which Medicare relies upon to establish payment levels for physicians' services.

${ }^{29}$ For further details, see Getz, Wenger, Campo et al. [2008].

${ }^{30}$ Very similar figures are obtained for the slightly larger set of protocols where the investigator contracts do not specify the number of patients at a site, or from a reweighting of the protocol-level statistics by the number of contracts per protocol.
} 
These changes in nominal TGPP, SWE, and number of patients per site suggest that important changes are occurring in the cost and nature of outsourced clinical research. Of course, some of these trends may reflect a changing composition of the sample in the mix of therapeutic areas and phases of research, and in the location of sites in the US versus other countries. Tables III through V provide descriptive statistics on the composition of the sample by various trial characteristics. Table III breaks out the fraction of observations annually by the development phase of the protocol. Clinical trials are conventionally categorized by stage of development. Phase I trials typically enroll a small number of healthy volunteers, and are focused on safety, tolerability, dose-ranging, pharmacokinetics, etc. Phase II trials enroll larger numbers of patients (not healthy volunteers) and investigate the potential for efficacy by assessing biological activity or effect of the treatment at alternative putatively safe doses. Phase III trials focus on efficacy of the treatment in therapeutic use, enrolling large numbers of patients. Phase IIIa refers to trials conducted prior to making a submission for regulatory approval, while Phase IIIb trials are those initiated after the submission for approval but prior to commercial launch. Phase IV trials are conducted after a drug has been approved, often as part of continued investigation of safety. While the fraction of the sample made up by sites involved in Phase I, Phase IIIb, and Phase IV studies was approximately stable, there has been a significant swing in the shares of Phase II and Phase IIIa. In 1989, Phase II trials made up less than $10 \%$ of the sample, and Phase IIIa almost $75 \%$. By the end of the sample period in 2011, Phase II studies comprised almost $30 \%$ of trials and Phase IIIa studies dropped to under $60 \%$. If early stage trials are more costly to conduct on a per patient basis, then this shift among trial phases may account for some of the increase in mean TGPP over time.

Table IV presents the allocation of investigator grants over 15 different therapeutic areas. Reflecting the burden of disease, trials involving the six "largest" therapeutic areas (central nervous system, cardiovascular, respiratory system, endocrine, oncology and anti-infectives) make up $70 \%$ of the sample on average. Shares of central nervous system and oncology trials grew somewhat over time until 2005-6, while cardiovascular shrank, suggesting that to the extent central nervous system and oncology trials are relatively more costly to conduct, these compositional changes may have some effect on increases in average TGPP up to 2005-2006.

Table V presents the geographic breakdown of the sites in this sample. Over the entire sample time period, $56 \%$ of sites were in the US, with most of the remainder in other OECD 
countries, and only 5.4\% in the rest of the world (ROW). Interestingly, although the US share is about $80 \%$ in both the earliest and latest years, there is substantial year-to-year and trend variability. However, as shown in Table I we observe considerably smaller numbers of observations in 1989-1991 and 2003-2011 relative to the 1992-2002 time period; any trend analysis is therefore tentative.

\section{Hedonic Price Index Methodology}

The hedonic pricing approach has a long tradition in economic measurement, going back almost a century. ${ }^{31}$ In essence, the hedonic approach treats the item being priced as a bundle of observed characteristics, and using multivariate regression methods, estimates "shadow prices" of each of the observed characteristics and the aggregate price index as a composite of the observed characteristics each multiplied by its shadow price. In practice, given observations in each period $t$ on the prices $\mathrm{P}_{\mathrm{it}}$ of a set of items $i$ with characteristics $\mathrm{X}_{\mathrm{it}}$, this means estimating a regression model on pooled data of the form $\log \left(\mathrm{P}_{\mathrm{it}}\right)=\mathrm{X}_{\mathrm{it}} \beta+\mathrm{Z}_{\mathrm{t}} \gamma+\varepsilon_{\mathrm{it}}$ where $\mathrm{Z}_{\mathrm{t}}$ is a set of dummy variables for each period and $\varepsilon_{\mathrm{it}}$ is a random error term. This semi-log functional form is widely used in hedonic price analysis. ${ }^{32}$ Predicted values from this regression provide the basis for computing changes in a "quality-adjusted" composite price index $\mathrm{P}_{\mathrm{t}}$ : with a set of time dummies in the regression, the change in the composite index relative to the base period is given by the exponentiated values of their estimated coefficients $(\hat{\gamma})$. Although $\mathrm{E}[\exp (\mathrm{P})] \neq \exp (\mathrm{E}[\mathrm{P}])$ and $\varepsilon_{\text {it }}$ may not be homoscedastic, suggesting a "smearing" adjustment of the type discussed in the medical costs literature, ${ }^{33}$ with time dummies in the regression these adjustment factors will typically be small. ${ }^{34}$ (In the case where residuals are homoscedastic within time periods but heteroscedastic across time, adjustments such as the nonparametric method proposed by Duan

\footnotetext{
${ }^{31}$ For an historical overview of hedonic price analysis, see ch. 4 in Ernst R. Berndt, The Practice of Econometrics: Classic and Contemporary, Reading, MA: Addison-Wesley Publishing Company, 1991 also see Ernst R. Berndt, Zvi Griliches and Neal J. Rappapor [1995]; and Berndt and Rappaport [2001], "Price and Quality of Desktop and Mobile Personal Computers: A Quarter Century Historical Overview”, American Economic Review 91(2):268-273, May..

${ }^{32}$ See Berndt [1991], ch. 4, and Jack E. Triplett, Handbook on Hedonic Indexes and Quality Adjustment in Price Indexes, Paris, Organization for Economic Community Development, 2006 for further discussion.

${ }^{33}$ Applications are primarily in modeling health care costs and outcomes. See, for example, Nathan Duan "Smearing Estimate: A Nonparametric Retransformation Method", Journal of the American Statistical Association 78:605-10, 983; Willard G. Manning and John Mullahy, "Estimating log mod els: To Transform or Not to Transform?", Journal of Health Economics 20:461-94, 2001; Willard G. Manning, "The Logged Dependent Variable, Heteroskedasticity, and the Retransformation Problem", Journal of Health Economics 17:283-95, 1998; and John Mullahy, "Much Ado About Two: Reconsidering Retransformation and the Two-Part Model in Health Econometrics, Journal of Health Economics 17:247-81, 1998.

${ }^{34}$ See Triplett [2006] p.34, footnote 41.
} 
will give estimates that are numerically identical to non-adjusted ones. ${ }^{35} \mathrm{We}$ found very similar adjusted and unadjusted estimated index values, and here we report only estimates with no further adjustment for cross-year heteroscedasticity.)

In this application, the "priced item" is the investigator total grant cost per patient. TGPP, and our hedonic regression takes the form $\log \left(\mathrm{TGPP}_{\mathrm{it}}\right)=\mathrm{X}_{\mathrm{it}} \beta+\mathrm{Z}_{\mathrm{t}} \gamma+\varepsilon_{\mathrm{it}}$, with $\mathrm{X}$ containing site and trial characteristics including planned number of patients at the investigator's site, location and number of sites and countries participating in the trial, phase of development, therapeutic area, and the site work effort (SWE) measure of trial burden and complexity. ${ }^{36} \mathrm{Z}_{\mathrm{t}}$ are annual indicator variables. Estimated standard errors are Huber-White robust, clustered by trial protocol; computations were carried out in STATA.

\section{Estimation and Price Index Results}

We now report results based on various regressions, and calculate corresponding average annual growth rates (AAGRs) of implied hedonic price indexes. Although all regressions have as regressors indicator variables for therapeutic class and year, we pool over and then run separate regressions by trial phase; in terms of time periods, we pool over the entire 1989-2011 time period, and then run separate regressions for the 1989-1999 and 2000-2011 subperiods. ${ }^{37}$ In all cases the dependent variable is the logarithm of total grant cost per patient (ln TGPP). ${ }^{38}$

Of particular interest to us are the coefficient estimates on two clinical trial characteristics variables - the logarithm of number patients at the site (LPATIENTS) and site work effort $(\mathrm{SWE}){ }^{39}$ Note that we have no expectation regarding the sign of the coefficient on the LPATIENTS variable; a negative estimate implies economies of scale at the site level, whereas a positive estimate corresponds to diseconomies of scale. Because SWE measures the cumulative burden of various clinical trial protocol procedures, we expect it to have a positive coefficient.

\footnotetext{
${ }^{35}$ See Duan reference in footnote 33 above.

${ }^{36}$ Using $\log (\mathrm{SWE})$ does not change the sign or significance of the estimated SWE-related coefficient, or result in material differences in the other estimates.

${ }^{37}$ In all models, tests of the joint null hypothesis that coefficient estimates on SWE, LPATIENTS and the various indicator variables were stable over the two time intervals were decisively rejected.

${ }^{38}$ When pooled over time, the number of observations in the top panel regressions is 207,950 (All), 118,477 (US Only), and 89,473 (Rest of World); for the 1989-1999 (2000-2009) regressions, the corresponding numbers of observations are $125,736(82,217), 66,246(52,231)$ and 59,490 $(29,983)$. Of course the number of observations in the various by phase regressions is smaller, with the smallest number being 2,738 for the 2000-2011 Phase I regressions.

${ }^{39}$ The log transform is used for patients because of the high degree of skewness and wide range of this variable. SWE falls in a much tighter range. No substantial differences in the results were obtained using $\log (\mathrm{SWE})$.
} 
Parameter estimates on these two variables, under alternative models and time periods, are presented in Table VI. With two exceptions (both involving LPATIENTS in Phase II trials), all of the estimated coefficients are statistically significant at the $1 \%$ level, based on robust standard errors.

A number of results are striking. As shown in the top panel of Table VI, when pooled over all phases (but including trial phase as indicator variables), globally and for ex-US (Rest of World), in all three time period regressions the estimated coefficient on LPATIENTS is positive and highly significant; however, for the US Only model the coefficient estimate is negative and significant. The implied estimated elasticities of TGPP with respect to patients range from -0.122 to 0.183 .

The pattern of estimates on LPATIENTS becomes a bit more nuanced when separate regressions are run by trial phase. Specifically, a general pattern that prevails is that negative estimates occur for the Phase I and Phase II regressions for the All and Rest of World regressions, but these estimates become positive and ever larger as one moves to the increasingly larger patient size trials. In Phase IIIA, Phase IIIB and Phase IV trials, almost all the estimates are positive and significant even at p-values <0.01. Also notable is the substantial range in estimates of the elasticity of TGPP with respect to patients, from -0.176 to 0.320 in the pooled 1989-2011 regressions, even larger from -0.219 to 0.305 in the 1989-1999 regressions, and in the 2000-2011 regressions, ranging from -0.190 to 0.272 . The pooled phases and by phase regressions based on US only data reveal considerably greater stability, both across the various time period regressions and across trial phases.

A second set of striking findings in Table VI is that every one of the estimates on the SWE variable is positive and statistically significant at $\mathrm{p}$-values $<0.01$, with the general (but not quite universal) pattern being that the positive estimates increase monotonically as one moves from the small Phase I to the larger Phase IIIB and Phase IV trials. The steepness of the positive slope with larger trial phase is flatter for the US Only regressions, however, than for the All and Rest of World regressions, with the Phase IV All and Rest of World estimates being particularly large; the vast majority of estimates on the SWE variable are in the range of 0.01 to 0.03 . Using a mean value of SWE of about 25 (see Table II), a one-unit increase in SWE changes it by about $4 \%(1 / 25)$, leading to about on average a $2 \%$ increase in TGPP, suggesting an elasticity of TGPP 
with respect to SWE of about $0.50(=0.02 / 0.04)$ when evaluated at the sample means. That is a very substantial effect.

A third implication of findings in Table VI is that they help explain factors affecting increases in trial costs, particularly for the US. The regression results suggests that increases in TGPP over time have been driven by increases in SWE and decreases in the number of patients at each site (particularly in the US where for each phase coefficient estimates on LPATIENTS are mostly negative.) Whether the changing composition among trial phases (towards Phase II and away from Phase IIIA - see Table III) can "explain" the increase in TGPP merits further examination.

We now move on to consider implications of these various regression models for the growth rate of our price indexes. As discussed above, annual values of an hedonic price index can be constructed from estimated coefficients on indicator variables by year. We summarize the growth rate of this index by computing the Annual Average Growth Rate (AAGR), which is the mean of year-on-year percentage changes in the index values. ${ }^{40}$ In the top panel of Table VII we report estimates of AAGRs in a "base" hedonic model that excludes our two prominent quality measures, namely, LPATIENTS and SWE, which from Table VI we have observed as being highly statistically significant. To quantify the importance of including these trial site quality characteristics in our hedonic regression equation, we compare AAGRs of predicted In TGPP with and without the LPATIENTS and SWE variables included by examining the relative growth of coefficients on the yearly indicator variables. The results are quite striking. With the pooled 1989-2011 regression, relative to the base model, TGPP grows much more slowly when the trial site characteristics are included $-4.31 \% / 6.96 \%$ for All (38\% lower AAGR), 3.62\%/7.01\% for US Only (48\% smaller AAGR), and 6.05\%/8.70\% for Rest of World (30\% lower AAGR). For the 1989-1999 regressions (second last column), the corresponding percent reductions in AAGRs are more modest - 6\% All, 31\% US Only, and 19\% for Rest of World, but for the most recent 2000-2011 time period regressions (last column), they are not only large proportionately-40\% lower AAGR for All, 56\% for US Only, and $28 \%$ for Rest of World, but the absolute differences in AAGRs are substantial as well-3.95\% (9.93\%-5.98\%) for All, 3.52\% (12.36\% - 8.84\%) for Rest of the World, and 4.10\% (7.38\% - 3.28\%) for the US

\footnotetext{
40 These arithmetic AAGRs were more stable than were estimates of compounded AGRs, since the latter were sensitive to choice of initial and end-year time periods.
} 
Only. We conclude, therefore, that controlling for the clinical trial quality characteristics LPATIENTS and SWE results in much lower AAGRs, and helps explain in part why it is that TGPP has been increasing steadily over the last two decades.

The bottom three panels of Table VII report AAGRs based on separate regressions by trial phase. As seen in the first column of Table VII based on pooled 1989-2011 regressions, over all trial phases US Only AAGRs are smaller than the All regression AAGRs, with the Rest of World regression AAGRs being greater than those in All for Phases I, II, Phases IIIA, and IIIB, but less than All for Phase IV. The variation in AAGRs within each set of regressions is quite large - from $3.78 \%$ to $11.91 \%$ in All, and $6.29 \%$ to $15.51 \%$ in the Rest of World, but only $3.48 \%$ to $6.78 \%$ in US Only regressions. AAGRs are generally lowest in Phase IIIA and IIIB, and mostly highest in Phase II and Phase IV. Even though the regressions involve pooled 19892011 data, as seen in the second and third column there is considerable variation across the two time intervals within the pooled regression, though less in US only than in the All and Rest of World regressions.

Comparing 1989-1999 AAGRs from the pooled regression (column two) with those from the separate 1989-1999 regression (column four), and the 1999-2011 AAGRs from the pooled regression (column three) with those from the separate 2000-2011 regression (last column) provides some evidence regarding parameter stability. The 1989-1999 relative rankings of AAGRs across trial phases in columns two and four is quite robust, but slightly less so when comparing relative rankings in columns three and five. Particularly notable is the uniformly greatest growth rate during the 2000s in Phase IV trials, with substantial but less uniformly large AAGRs in Phase I studies.

Of particular interest is a comparison of growth rates of price indexes derived from parameters of the base hedonic model including indicator variables for trial phase, therapeutic area, and year, the augmented hedonic model with SWE and LPATIENTS added to the base hedonic model as regressors, and the BRDPI published by the NIH under contract to BEA (the last based on only NIH funded research, and performed primarily but not exclusively at academic medical centers). In Figure 3 we plot the annual time series of the three price indexes, based on US-only regression estimates for the two hedonic equations, with the normalized series for mean TGPP included for reference. The results are striking. Indexed to 1.000 in 1989, in 2011 the BRDPI is 2.205, very close to the augmented hedonic price index value of 2.138; with 
very similar AAGRs and compound annual growth rates (CAGRs) over the period: $3.66 \%$ AAGR and CAGR for the BRDPI and 3.63\% AAGR and 3.31\% CAGR for the augmented hedonic index. By contrast, the 2011 price index derived from the base hedonic model (omitting the SWE and LPATIENTS quality variables) has a value of 4.178, and an AAGR of $7.01 \%$ and CAGR of $6.71 \%$. Thus the input cost based BRDPI and augmented hedonic model price indexes grow much more slowly - at about one half of the rate as those derived from the base hedonic model, which controls only for changes in the "mix" of the sample over trial phases and therapeutic classes.

Tables VIII and IX present results of two sets of exploratory findings. Although issues of sample size are likely to become important, we estimate ln TGPP equations at the level of the therapeutic class, pooled 1989-2011 and separately for 1989-1999 and 2000-2011; as before we do three geography-based estimations-All, US-Only and Rest of World. There are 15 therapeutic classes in our trial data, 14 of them involving biopharmaceuticals plus a devices and diagnostics category. In Table VIII we report AAGRs by therapeutic class for the US-Only and Rest of World regressions; note that because of the absence of any observations in some years, there is some variability from the 1989-2011, 1989-99 and 2000-11 beginning and ending years, as is described at the bottom of the table. The most striking feature of Table VIII is the substantial variability in the AAGRs; not shown is the even greater variability in the estimates on the year indicator variables within each therapeutic class. While is it likely that there is in fact substantial variation across therapeutic classes in the rate of change of trial costs, at least some of the variation is likely attributable to the smaller sample sizes in certain years that result from disaggregating into 15 therapeutic classes. This makes it difficult to estimate the hedonic index values precisely, and particularly outside the US there are only enough observations for some therapeutic classes to estimate the index values in a limited number of years.

We conclude that constructing price indexes for clinical trials at the level of therapeutic classes (in our case, which number 15) is likely to be infeasible because of sample size issues, particularly for ex-US sites.

Our final exploratory price index analysis involves aggregating up from individual multiple sites within a given trial to the trial level at which there is a common protocol. This allows us to examine whether number of trial sites and the geographical scope of the sites affects our dependent variable, ln TGPP. This aggregation reduces our sample size from the 207,950 
sites in Table VI to 24,172 distinct trials. We construct two new variables that vary at the level of the individual trial protocol: number of sites, and number of sites per country. We also recalculate the dependent variable, LPATIENTS and the SWE variable at the trial level of aggregation. We do not have a prior expectation regarding the sign of the coefficient on total number of sites per trial. This coefficient will capture whether or not there are cost impacts of allocating a given number of patients across different numbers of sites. To the extent there are fixed costs incurred at each site for setting up patient recruitment, independent of the number of patients enrolled at a given site, then holding the numbers of trial patients constant, the aggregate TGPP would be expected to increase with the number of sites. On the other hand, if fixed costs are largely trial specific rather than site-specific, and are carried in the "overhead" part of trial costs which we do not observe in these data, then they will either not affect site-level costs, i.e. no observable impact of number of sites on aggregate TGPP, or to the extent that they reduce site-specific costs otherwise borne by investigators, will result in a negative relationship between aggregate TGPP and number of sites.

Some of these trial-level fixed costs are likely to be country-specific, reflecting factors such as national institutional review boards, import duties and tariffs, medical licensing conventions or other costs of conforming to a given country's regulatory framework and medical infrastructure. To the extent that these costs are "pushed down" to individual sites, rather than absorbed in the overall "overhead" cost of the trial then aggregate TGPP may be affected by the number of sites per country. We therefore also control for each trial's number of sites per country.

In Table IX, we report coefficient estimates on the number of sites, and the number of sites per country, for regressions at two levels of aggregation: Pooled over phases (but with phase indicator variables included as regressors), and separately by trial phase. ${ }^{41}$ When pooled over phases, the estimate on number of sites is positive and strongly significant, while the number of sites per country is negative but not significant. When estimated separately by phase, signs on the number of sites variable are mixed but monotonically decline moving from early to late phases, although none is statistically significant. However, all but one of the estimates on the number of sites per country are positive, and statistically significant in the case of Phase II

\footnotetext{
${ }^{41}$ SWE, LPATIENTS, and year, therapeutic class and phase indicators are also included in the regressions. Estimated coefficients on SWE and LPATIENTS were similar in magnitude to those obtained in the site-level regressions, and statistically significant at the $1 \%$ level.
} 
and IIIA trials. In almost all cases, however, the absolute magnitudes of the coefficient estimates are very small — an order of magnitude or smaller than those on the LPATIENTS and SWE variables reported in Table VI. We conclude that at the level of a clinical trial protocol, the number of sites and number of sites per country do not appear to have a material effect on the total grant cost per patient. These trial characteristics might, however, have varying effects on the sponsors' overall "headquarters" overhead costs, which we do not observe.

\section{Alternate Index Methodologies}

One important practical issue with hedonic indexes such as those estimated in the previous section is that of updating and revisions: over time as new data are acquired, statistical agencies undertaking re-estimation of the pooled regression model for the expanded and updated data set will likely find there are changes in the estimated time dummy coefficients and thus in the historical hedonic price index values derived from them. Revising historical time series of price indexes each time a new time period is added is an unattractive feature of the pooled hedonic price index methodology. One way to avoid this problem is to construct an index based on results from a set of sequentially estimated "adjacent year" regressions: for any year t, estimate the model on data only for years $t$ and $t-1$, with the coefficient on a dummy variable for year $t$ providing an estimate of the change in a quality-adjusted or characteristics-adjusted index over the two periods. Year-on-year changes can be then be chained to create an index for the whole time period.

Focusing on the US-only sample, we find that indexes constructed using this method track the hedonic index estimated from the pooled sample quite closely. For the "base" model that controls only for phase and therapeutic class, the AAGR of the adjacent-years index is $7.47 \%$, compared to the $7.01 \%$ for the pooled hedonic (and $7.52 \%$ for the mean nominal TGPP). For the hedonic models that include SWE and LPATIENTS, the AAGR for the adjacent-year index is $3.73 \%$, compared to $3.62 \%$ for the pooled hedonic. (Recall that the AAGR for the BRDPI is $3.66 \%$.) These results reflect remarkably similar growth rates based on alternative price index methodologies over almost a quarter century time period. While a formal statistical test rejects equality of the coefficients on all the variables across all pairs of adjacent years, visual examination of the coefficients in each of the adjacent-year regressions (not presented here) shows them to be quite stable over time. 
An even more general alternative approach to estimating the constant-characteristics change in price (sometimes known as the Pure Hedonic method, see Pakes (2003), and Berndt, Griliches and Rappaport (1995)) is to estimate regression coefficients separately for each year, and then compute Paasche-style or Laspeyres-style index ratios using alternative fixed quantity (characteristic) weights.. For example, if $X_{i 0}$ are the characteristics for each observation in year 0 and $\beta_{0}$ are hedonic coefficients estimated from the year 0 regression, and $X_{i 1}$ and $\beta_{1}$ are the characteristics for each observation and hedonic coefficients estimated for year 1, then a Laspeyres-type measure of the change in constant-characteristics prices can be calculated as $L_{1}=\frac{\sum_{i} X_{i 0} \beta_{1}}{\sum_{i} X_{i 0} \beta_{0}}$ and the corresponding Paasche-type index as $P_{1}=\frac{\sum_{i} X_{i 1} \beta_{1}}{\sum_{i} X_{i 1} \beta_{0}}$, with the Fischer Ideal index given by $F_{1}=\sqrt{L_{1} P_{1}}$.

The advantage of this method over the adjacent-years dummy variable method is that in the adjacent year regressions the only parameters that is allowed to vary between the two adjacent years is the year dummy variable, whereas in the Pure Hedonic method all coefficient estimates (interpreted as shadow prices of the characteristics) can change between years. In the Pure Hedonic method, these yearly shadow prices are weighted by fixed weights - either the base period (Laspeyres) or the current period (Paasche) characteristics.

A potential problem with the chained $L_{1}$ and $P_{1}$ price indexes is "drift." If there is "bouncing" (upward changes followed by downward changes) of relative prices over a multiyear period, then negative autocorrelations in prices combined with negative correlations between price and quantity changes, can generate an upward "drift" in the chained $\mathrm{L}_{1}$ index and a downward "drift" in the $\mathrm{P}_{1}$ index. $^{42}$. Although the theoretical foundations have to the best of our knowledge not been derived, it is possible that a superlative chained Fisher Ideal index that is the geometric mean of a chained Laspeyres and chained Paasche index would embody offsetting drifts, thereby resulting in a more reliable chained index than either the Laspeyres or Paasche.

Results from calculating these indexes and chaining for all years in the US-only sample are broadly consistent with the results from the adjacent-years method. For the regression specification that includes SWE and LPATIENTS, the AAGR for a Laspeyres-type pure hedonic index was 5.40\%, 2.44\% for a Paasche-type pure hedonic index, and 3.89\% for the Fischer-Ideal

\footnotetext{
${ }^{42}$ For a discussion of drift in the context of alternative direct and chained index numbers, see Bohdan J. Szulc, "Linking Price Index Numbers", in W. Erwin Diewert and Claude Montmarquette, eds., Price Level Measurement: Proceedings from a conference sponsored by Statistics Canada, Ottawa, Canada: Ministry of Supply and Services Canada, December 1983, pp. 537-566.
} 
pure hedonic index. We plot the various hedonic indexes (based on the augmented regression) in Figure 4. There it is seen that the pooled hedonic, adjacent-years hedonic and pure hedonic Fisher Ideal indexes track each other (and the BRDPI) quite closely. Values of these four indexes are remarkably close to each other in 2011: 2.046 for the pooled hedonic, 2.138 for the adjacent-years hedonic, and 2.221 for the pure hedonic Fisher-Ideal, compared to 2.205 for the BRDPI. These four indexes have very similar AAGRs $(3.62 \%, 3.73 \%, 3.89 \%$, and 3.66\%, respectively) and CAGRs $(3.31 \%, 3.52 \%, 3.69 \%$, and 3.66\%, respectively. In contrast, the chained pure hedonic Paasche (with potential downward drift) and the chained pure hedonic Laspeyres (with possible upward drift) have 2011 index values well below and above the other four. The Paaache index value for 2011 was only 1.627 (AAGR of 2.4\% and CAGR of 2.24\%) while the Laspeyres was 3.033 (AAGR of $5.40 \%$ and CAGR of $5.17 \%$ ).

The close correspondence between the results for all three of hedonic index methods (pooled time dummy, adjacent-years time dummy, and "pure hedonic") suggest to us that the underlying relationship between the observed nominal transaction prices and the key characteristics of each contract (SWE, LPATIENTS, phase and therapeutic class) is quite robust, at least within the US-only sample. It would therefore appear to be reasonable to develop a periodically updated index using either the pure hedonic Fisher Ideal or adjacent-year time dummy methods with US data,

\section{Summary, Conclusions, Limitations and Future Research}

Expenditures on clinical trials undertaken to develop new drugs have increased dramatically over the past 30 years. To better understand the underlying causes it is critical to be able to decompose increases in total spending into the "price effect", the "quantity effect," and the "quality" effect. Are biopharmaceutical companies doing more clinical research, has the cost of doing a given amount of research increased, or are both occurring? In this study we focus on the "unit costs" of certain aspects of conducting clinical trials. These have risen substantially in recent decades, outpacing general inflation and other measures of changes in costs of other inputs to biomedical R\&D. Our results suggest that these increases in trial costs are not solely attributable to changes in input costs such as wages, equipment, and facilities. They also appear to have been driven to a substantial extent by two other phenomena: smaller numbers of patients per site, and increases in the "effort" level required by investigators as study protocols have required more costly and complex monitoring and testing of subjects. Evaluated at the sample 
means, our estimated elasticity of TGPP with respect to SWE is about 0.5. Over the 1989-2011 time period, mean values of SWE have varied by as much as a factor more than three, generally rising but not quite monotonically over time (Table I). This suggests that increased trial intensity and trial design complexity — as measured by SWE — have been major drivers of the increase in nominal TGPP, over and above changes in input costs. By comparison, our estimates of the elasticity of TGPP with respect to patients per site is much smaller in absolute magnitude (about -0.120 for the US, see Table VI), and since the relative decrease in patients over time is smaller than the relative increase in SWE, declining site "size" is not as large a driver of TGPP increases as are changes in SWE. While the trends in SWE and patients per site are in turn likely driven to some extent by cost differences across therapeutic classes and phases of clinical development, the effects we find are estimated controlling for such study characteristics, and are not just an artifact of changes in the composition of the sample. The size of the effects that we find implies that any effort to track costs of clinical research should pay close attention to the nature of study protocols and the organization and management of trials. The extent to which increases in SWE are attributable to increased regulatory requirements and scrutiny versus evolving commercial product differentiation strategies is unknown and merits further analysis. Our findings point to the value of using the hedonic regression methodology in this context.

The price indexes for commercial clinical research constructed here appear to behave remarkably similar to those computed by BEA on behalf of NIH for input costs for public sector biomedical R\&D, once one controls for SWE and LPATIENTS in the commercial trials; this unexpected congruence merits more careful attention and confirmation by government statistical agencies and other entities with an interest in tracking R\&D costs in this sector. Specifically, the AAGR of a price index that controls only for therapeutic class and phase of development grows almost twice as fast as the NIH BRDPI input costs index. Interestingly, once the scale of investigator/site activity and the effort required by a study protocol are also controlled for, the estimated "quality-adjusted" rate of inflation within the US is remarkably similar to the BRDPI. This suggests that increases in commercial clinical trial costs are driven primarily by changes in the nature of clinical research rather than by inflation in input costs.

Commercial databases such as the one we have used here appear to have great potential as a source of data for such price index measurement purposes, particularly if restricted to the US context. Using these data it would appear to be feasible to reliably compute measures of price 
inflation for this aspect of clinical research, to do this separately for different phases of clinical development, and for some but not all therapeutic classes. The geographic reach of these data sources also presents interesting opportunities to benchmark $R \& D$ costs across different regions within the US, as well as across countries. However, care needs to be taken to ensure that the number of observations is adequate in each of the annual therapeutic class data cells.

There are some important limitations to this study. In particular, we look only at one component of trial costs: payments to clinical investigators. In this dataset, these account for about one half of the total cost of a trial. It may well be that some of the higher per-patient costs created by having fewer patients per site and increased effort required by the protocol are offset by savings in the sponsors' headquarters' costs of centralized administration and coordination of trials that we do not observe here. Limited availability of data prevents us from drawing strong conclusions about trends in total trial costs and underlying factors in recent years. As noted above, the prices we observe are only for the payments made by the trial sponsor to investigators, either directly or through a CRO, and additional costs for trial design and administration are not included. To the extent these additional "overhead" costs are understood as internal headquarters costs of the entity purchasing the R\&D output of trial sites, then our focus on investigator contracts has the appropriate scope for purposes of developing a PPI for contracted R\&D services. On the other hand, if costs of trial design and administration are understood as R\&D costs, then our focus may be too narrow. In many instances, and increasingly over time, trial sponsors contract with a CRO to perform some or all of these functions, and such payments may well be considered part of the contracted R\&D services. This is an issue that warrants further investigation, but would require additional data on the nature of payments by trial sponsors to CROs, and development of a linkage between these contracts and the investigator payments analyzed here. In this context it is unclear how well the measure of "site work effort" used here captures differences in the burden imposed by, for example, running more complex trial protocols, where some of the "overhead" associated with management and administration is being borne by the site as opposed to the sponsor or CRO, as distinct from increased use of more costly interventions or methods of measuring trial endpoints.

A second limitation of this study is that we cannot evaluate how representative is the universe of clinical trial contracts in the Medidata sample — over time, across therapeutic classes, and geographically. Lastly, since the identity of study sponsors and investigators was not 
available to us, we were not able to investigate differences in costs across (for example) trials sponsored by large versus small commercial entities, or where public sector or non-profit organizations are involved as sponsors or investigators rather than industry.

We look forward to addressing these questions in future research. 
Table I: Total Grant Cost per Patient (TGPP)

\begin{tabular}{ccccc}
\hline year & mean & p50 & sd & $\mathrm{N}$ \\
& & & & \\
1989 & 3772.59 & 2779.43 & 2921.34 & 1370 \\
1990 & 4385.77 & 3147.77 & 4016.57 & 3443 \\
1991 & 3774.43 & 2774.83 & 4186.06 & 9288 \\
1992 & 3493.63 & 2399.00 & 6129.31 & 14126 \\
1993 & 3664.58 & 2325.45 & 4254.28 & 15733 \\
1994 & 3911.39 & 2882.35 & 3925.46 & 16625 \\
1995 & 4183.00 & 3203.85 & 3941.90 & 15670 \\
1996 & 4884.89 & 3748.77 & 4708.14 & 14442 \\
1997 & 4549.12 & 3200.00 & 4422.39 & 13321 \\
1998 & 5393.70 & 3948.38 & 5445.89 & 14370 \\
1999 & 5501.08 & 4361.94 & 4874.07 & 13943 \\
2000 & 6220.42 & 4682.79 & 6243.02 & 18671 \\
2001 & 6078.96 & 4777.00 & 5150.11 & 16864 \\
2002 & 6567.58 & 4744.10 & 5984.32 & 12201 \\
2003 & 8147.90 & 6765.00 & 6866.55 & 6515 \\
2004 & 10264.00 & 8582.72 & 7758.22 & 3216 \\
2005 & 11412.77 & 9682.02 & 7828.89 & 2693 \\
2006 & 12364.68 & 10900.00 & 7460.17 & 4012 \\
2007 & 13001.19 & 10738.47 & 8863.90 & 4764 \\
2008 & 14834.64 & 12720.94 & 10328.42 & 3216 \\
2009 & 16518.28 & 13965.42 & 12550.80 & 4591 \\
2010 & 15099.19 & 12581.93 & 10860.27 & 4814 \\
2011 & 16566.55 & 13222.14 & 13556.92 & 2188 \\
& & & & \\
Total & 6191.80 & 4195.07 & 6860.91 & 216076 \\
& & & & \\
\hline
\end{tabular}


Table II: SWE and Patients per Sitge

\begin{tabular}{|c|c|c|c|c|c|c|c|c|}
\hline \multirow[b]{2}{*}{ YEAR } & \multicolumn{4}{|c|}{$\begin{array}{c}\text { (a) Site Work Effort (SWE) } \\
\text { (Protocol level) }\end{array}$} & \multicolumn{4}{|c|}{$\begin{array}{l}\text { (b) Patients } \\
\text { (Site level) }\end{array}$} \\
\hline & MEAN & MEDIAN & STD. DEV. & $\mathrm{N}$ & MEAN & MEDIAN & STD. DEV. & $\mathrm{N}$ \\
\hline 1989 & 17.07 & 13.29 & 12.88 & 240 & 25.48 & 20.00 & 33.2476 & 1370 \\
\hline 1990 & 16.94 & 13.44 & 13.38 & 512 & 21.26 & 15.00 & 27.3178 & 3443 \\
\hline 1991 & 14.85 & 11.16 & 12.11 & 1280 & 20.64 & 15.00 & 32.4249 & 9288 \\
\hline 1992 & 17.74 & 13.46 & 15.45 & 1833 & 18.50 & 12.00 & 25.253 & 14126 \\
\hline 1993 & 18.92 & 13.66 & 17.79 & 2024 & 17.04 & 12.00 & 24.5758 & 15733 \\
\hline 1994 & 21.58 & 16.22 & 18.20 & 1967 & 16.37 & 12.00 & 24.6318 & 16625 \\
\hline 1995 & 23.41 & 18.11 & 21.20 & 1826 & 14.39 & 10.00 & 26.52 & 15675 \\
\hline 1996 & 23.69 & 18.64 & 19.32 & 1791 & 14.83 & 10.00 & 36.2925 & 14444 \\
\hline 1997 & 24.93 & 19.26 & 20.11 & 1540 & 14.14 & 10.00 & 17.6792 & 13321 \\
\hline 1998 & 25.77 & 19.64 & 19.81 & 1495 & 14.60 & 10.00 & 24.9971 & 14370 \\
\hline 1999 & 26.87 & 20.55 & 23.89 & 1369 & 13.85 & 10.00 & 17.0209 & 13944 \\
\hline 2000 & 28.18 & 22.44 & 22.47 & 1890 & 11.27 & 9.00 & 14.7171 & 18696 \\
\hline 2001 & 29.91 & 22.78 & 24.18 & 1920 & 12.11 & 10.00 & 13.8476 & 16907 \\
\hline 2002 & 28.91 & 21.97 & 24.06 & 1389 & 12.07 & 10.00 & 12.2206 & 12250 \\
\hline 2003 & 35.46 & 29.29 & 27.26 & 933 & 13.30 & 10.00 & 18.5263 & 6629 \\
\hline 2004 & 43.57 & 34.33 & 34.09 & 569 & 12.18 & 9.00 & 14.5193 & 3362 \\
\hline 2005 & 47.65 & 39.06 & 38.04 & 307 & 10.73 & 10.00 & 14.041 & 2784 \\
\hline 2006 & 45.53 & 35.62 & 35.96 & 274 & 11.80 & 10.00 & 9.62717 & 4027 \\
\hline 2007 & 37.71 & 30.58 & 22.10 & 227 & 10.30 & 9.00 & 8.47037 & 4784 \\
\hline 2008 & 48.64 & 41.55 & 37.49 & 183 & 11.10 & 7.00 & 19.9492 & 3291 \\
\hline 2009 & 48.98 & 41.76 & 33.86 & 263 & 11.81 & 8.00 & 15.4748 & 4729 \\
\hline 2010 & 46.16 & 38.52 & 33.39 & 275 & 11.79 & 8.00 & 23.6707 & 4907 \\
\hline 2011 & 45.69 & 41.31 & 30.59 & 129 & 11.83 & 8.00 & 17.0184 & 2224 \\
\hline Total & 25.94 & 19.21 & 23.28 & 24236 & 14.42 & 10.00 & 22.58 & 216929 \\
\hline
\end{tabular}




\section{Table III: Development Phase Percentages}

\begin{tabular}{cccccc}
\hline Year & Phase I & Phase II & Phase IIIA & Phase IIIB & Phase IV \\
& & & & & \\
1989 & $2.12 \%$ & $9.12 \%$ & $72.55 \%$ & $6.35 \%$ & $9.85 \%$ \\
1990 & $3.08 \%$ & $13.94 \%$ & $69.53 \%$ & $5.84 \%$ & $7.61 \%$ \\
1991 & $3.45 \%$ & $15.13 \%$ & $50.67 \%$ & $12.75 \%$ & $18.01 \%$ \\
1992 & $3.58 \%$ & $14.14 \%$ & $58.47 \%$ & $6.98 \%$ & $16.82 \%$ \\
1993 & $3.96 \%$ & $18.34 \%$ & $56.79 \%$ & $4.74 \%$ & $16.16 \%$ \\
1994 & $3.70 \%$ & $18.80 \%$ & $58.69 \%$ & $7.65 \%$ & $11.16 \%$ \\
1995 & $4.20 \%$ & $20.52 \%$ & $54.23 \%$ & $6.63 \%$ & $14.41 \%$ \\
1996 & $4.84 \%$ & $23.09 \%$ & $52.94 \%$ & $8.55 \%$ & $10.58 \%$ \\
1997 & $4.14 \%$ & $18.08 \%$ & $56.53 \%$ & $8.85 \%$ & $12.40 \%$ \\
1998 & $3.63 \%$ & $19.08 \%$ & $49.45 \%$ & $13.84 \%$ & $13.99 \%$ \\
1999 & $4.06 \%$ & $20.35 \%$ & $49.25 \%$ & $14.08 \%$ & $12.26 \%$ \\
2000 & $3.39 \%$ & $17.59 \%$ & $49.86 \%$ & $17.72 \%$ & $11.44 \%$ \\
2001 & $3.64 \%$ & $16.60 \%$ & $50.21 \%$ & $14.95 \%$ & $14.60 \%$ \\
2002 & $3.00 \%$ & $20.99 \%$ & $46.78 \%$ & $16.56 \%$ & $12.68 \%$ \\
2003 & $4.06 \%$ & $17.79 \%$ & $45.26 \%$ & $16.52 \%$ & $16.38 \%$ \\
2004 & $4.49 \%$ & $23.38 \%$ & $42.15 \%$ & $18.59 \%$ & $11.39 \%$ \\
2005 & $2.33 \%$ & $26.08 \%$ & $51.33 \%$ & $9.05 \%$ & $11.21 \%$ \\
2006 & $3.25 \%$ & $15.50 \%$ & $69.23 \%$ & $3.35 \%$ & $8.67 \%$ \\
2007 & $2.17 \%$ & $25.90 \%$ & $49.85 \%$ & $15.49 \%$ & $6.58 \%$ \\
2008 & $2.98 \%$ & $23.06 \%$ & $48.40 \%$ & $16.38 \%$ & $9.18 \%$ \\
2009 & $3.45 \%$ & $23.41 \%$ & $58.64 \%$ & $7.76 \%$ & $6.75 \%$ \\
2010 & $2.45 \%$ & $31.97 \%$ & $46.06 \%$ & $11.98 \%$ & $7.54 \%$ \\
2011 & $1.66 \%$ & $25.99 \%$ & $55.35 \%$ & $6.74 \%$ & $10.25 \%$ \\
& & & & & \\
Total & $3.67 \%$ & $19.26 \%$ & $53.07 \%$ & $11.18 \%$ & $12.83 \%$ \\
& & & & &
\end{tabular}

Table entries are the fraction of investigator contracts in that year for studies at each phase of clinical development. Based on 216,929 total observations. 
Table IV: Distribution of Sample by Therapeutic Class

\begin{tabular}{|c|c|c|c|c|c|c|c|}
\hline Year & $\begin{array}{c}\text { Anti- } \\
\text { Infective }\end{array}$ & $\begin{array}{l}\text { Cardio- } \\
\text { vascular }\end{array}$ & $\begin{array}{c}\text { Central } \\
\text { Nervous } \\
\text { System }\end{array}$ & Dermatology & $\begin{array}{l}\text { Devices and } \\
\text { Diagnostics }\end{array}$ & Endocrine & $\begin{array}{c}\text { Gastro- } \\
\text { intestinal }\end{array}$ \\
\hline 1989 & $7.66 \%$ & $14.82 \%$ & $14.38 \%$ & $4.09 \%$ & $0.00 \%$ & $3.28 \%$ & $10.07 \%$ \\
\hline 1990 & $9.09 \%$ & $21.64 \%$ & $9.70 \%$ & $2.27 \%$ & $0.20 \%$ & $7.29 \%$ & $9.00 \%$ \\
\hline 1991 & $8.81 \%$ & $18.62 \%$ & $14.69 \%$ & $2.45 \%$ & $0.12 \%$ & $6.01 \%$ & $13.18 \%$ \\
\hline 1992 & $8.10 \%$ & $16.69 \%$ & $15.33 \%$ & $2.63 \%$ & $0.27 \%$ & $5.88 \%$ & $9.80 \%$ \\
\hline 1993 & $10.13 \%$ & $19.70 \%$ & $14.37 \%$ & $2.58 \%$ & $0.35 \%$ & $7.29 \%$ & $9.92 \%$ \\
\hline 1994 & $11.56 \%$ & $20.82 \%$ & $16.72 \%$ & $2.15 \%$ & $0.48 \%$ & $6.42 \%$ & $6.35 \%$ \\
\hline 1995 & $8.09 \%$ & $18.65 \%$ & $16.66 \%$ & $2.07 \%$ & $0.05 \%$ & $6.46 \%$ & $4.43 \%$ \\
\hline 1996 & $8.42 \%$ & $16.45 \%$ & $17.50 \%$ & $1.88 \%$ & $0.18 \%$ & $7.57 \%$ & $3.82 \%$ \\
\hline 1997 & $6.58 \%$ & $15.54 \%$ & $19.06 \%$ & $1.73 \%$ & $0.29 \%$ & $9.10 \%$ & $4.51 \%$ \\
\hline 1998 & $7.24 \%$ & $15.85 \%$ & $15.49 \%$ & $2.75 \%$ & $0.15 \%$ & $11.69 \%$ & $1.88 \%$ \\
\hline 1999 & $5.49 \%$ & $18.89 \%$ & $14.08 \%$ & $2.36 \%$ & $0.32 \%$ & $11.93 \%$ & $2.76 \%$ \\
\hline 2000 & $6.93 \%$ & $15.28 \%$ & $14.85 \%$ & $2.40 \%$ & $0.52 \%$ & $13.61 \%$ & $3.36 \%$ \\
\hline 2001 & $10.56 \%$ & $9.39 \%$ & $13.95 \%$ & $2.83 \%$ & $0.15 \%$ & $14.83 \%$ & $5.20 \%$ \\
\hline 2002 & $6.39 \%$ & $8.42 \%$ & $13.84 \%$ & $2.02 \%$ & $0.17 \%$ & $17.37 \%$ & $3.85 \%$ \\
\hline 2003 & $8.63 \%$ & $9.94 \%$ & $22.85 \%$ & $6.14 \%$ & $0.86 \%$ & $12.25 \%$ & $2.16 \%$ \\
\hline 2004 & $5.68 \%$ & $12.31 \%$ & $27.96 \%$ & $1.81 \%$ & $1.04 \%$ & $13.06 \%$ & $0.39 \%$ \\
\hline 2005 & $4.92 \%$ & $21.62 \%$ & $21.12 \%$ & $3.84 \%$ & $1.11 \%$ & $5.28 \%$ & $2.12 \%$ \\
\hline 2006 & $2.36 \%$ & $12.14 \%$ & $27.04 \%$ & $2.48 \%$ & $0.15 \%$ & $19.44 \%$ & $3.30 \%$ \\
\hline 2007 & $4.77 \%$ & $4.52 \%$ & $17.52 \%$ & $1.17 \%$ & $0.31 \%$ & $18.42 \%$ & $7.46 \%$ \\
\hline 2008 & $3.77 \%$ & $6.05 \%$ & $17.23 \%$ & $3.80 \%$ & $0.64 \%$ & $21.27 \%$ & $5.62 \%$ \\
\hline 2009 & $9.45 \%$ & $1.69 \%$ & $18.95 \%$ & $1.78 \%$ & $2.24 \%$ & $23.77 \%$ & $4.95 \%$ \\
\hline 2010 & $6.64 \%$ & $1.26 \%$ & $12.43 \%$ & $5.05 \%$ & $2.71 \%$ & $31.57 \%$ & $4.18 \%$ \\
\hline 2011 & $1.35 \%$ & $0.63 \%$ & $14.88 \%$ & $6.07 \%$ & $6.03 \%$ & $28.46 \%$ & $0.05 \%$ \\
\hline Total & $7.87 \%$ & $14.79 \%$ & $16.21 \%$ & $2.56 \%$ & $0.47 \%$ & $11.44 \%$ & $5.29 \%$ \\
\hline
\end{tabular}

Table entries are the fraction of investigator contracts in that year for studies in each therapeutic area. Based on 216,929 total observations 
Table IV (cont.): Distribution of Sample by Therapeutic Class

\begin{tabular}{|c|c|c|c|c|c|c|c|c|}
\hline Year & $\begin{array}{l}\text { Genitourinary } \\
\text { System }\end{array}$ & Hematology & $\begin{array}{l}\text { Immuno- } \\
\text { modulation }\end{array}$ & Oncology & $\begin{array}{c}\text { Ophthal- } \\
\text { mology }\end{array}$ & $\begin{array}{c}\text { Pain and } \\
\text { Anesthesia }\end{array}$ & $\begin{array}{l}\text { Pharmaco- } \\
\text { kinetics }\end{array}$ & $\begin{array}{c}\text { Respiratory } \\
\text { System }\end{array}$ \\
\hline 1989 & $10.51 \%$ & $0.58 \%$ & $18.76 \%$ & $5.84 \%$ & $1.68 \%$ & $1.61 \%$ & $1.68 \%$ & $5.04 \%$ \\
\hline 1990 & $5.14 \%$ & $1.95 \%$ & $7.75 \%$ & $8.51 \%$ & $8.19 \%$ & $0.46 \%$ & $1.54 \%$ & $7.26 \%$ \\
\hline 1991 & $5.33 \%$ & $0.39 \%$ & $8.57 \%$ & $4.94 \%$ & $2.57 \%$ & $1.07 \%$ & $2.02 \%$ & $11.24 \%$ \\
\hline 1992 & $8.58 \%$ & $0.42 \%$ & $6.26 \%$ & $5.12 \%$ & $0.68 \%$ & $3.27 \%$ & $2.10 \%$ & $14.87 \%$ \\
\hline 1993 & $9.76 \%$ & $0.70 \%$ & $6.06 \%$ & $3.37 \%$ & $0.66 \%$ & $0.90 \%$ & $2.07 \%$ & $12.15 \%$ \\
\hline 1994 & $5.73 \%$ & $0.94 \%$ & $6.09 \%$ & $4.84 \%$ & $1.41 \%$ & $0.87 \%$ & $2.36 \%$ & $13.27 \%$ \\
\hline 1995 & $7.45 \%$ & $0.54 \%$ & $5.33 \%$ & $8.22 \%$ & $1.07 \%$ & $1.33 \%$ & $2.29 \%$ & $17.34 \%$ \\
\hline 1996 & $4.94 \%$ & $1.70 \%$ & $7.55 \%$ & $9.62 \%$ & $0.80 \%$ & $2.85 \%$ & $2.51 \%$ & $14.21 \%$ \\
\hline 1997 & $6.51 \%$ & $0.62 \%$ & $5.33 \%$ & $10.81 \%$ & $1.70 \%$ & $1.37 \%$ & $2.67 \%$ & $14.17 \%$ \\
\hline 1998 & $8.20 \%$ & $1.75 \%$ & $5.82 \%$ & $10.51 \%$ & $1.34 \%$ & $1.51 \%$ & $2.39 \%$ & $13.43 \%$ \\
\hline 1999 & $6.66 \%$ & $3.13 \%$ & $10.23 \%$ & $10.44 \%$ & $1.78 \%$ & $2.20 \%$ & $2.57 \%$ & $7.16 \%$ \\
\hline 2000 & $5.60 \%$ & $3.29 \%$ & $7.05 \%$ & $11.26 \%$ & $0.59 \%$ & $2.12 \%$ & $1.87 \%$ & $11.27 \%$ \\
\hline 2001 & $7.58 \%$ & $1.41 \%$ & $9.30 \%$ & $10.46 \%$ & $1.20 \%$ & $2.98 \%$ & $1.87 \%$ & $8.28 \%$ \\
\hline 2002 & $9.82 \%$ & $1.89 \%$ & $9.63 \%$ & $10.21 \%$ & $1.52 \%$ & $4.58 \%$ & $1.56 \%$ & $8.73 \%$ \\
\hline 2003 & $4.89 \%$ & $2.07 \%$ & $6.46 \%$ & $10.47 \%$ & $1.28 \%$ & $4.16 \%$ & $1.89 \%$ & $5.96 \%$ \\
\hline 2004 & $8.36 \%$ & $3.84 \%$ & $9.52 \%$ & $10.14 \%$ & $0.54 \%$ & $0.27 \%$ & $2.23 \%$ & $2.86 \%$ \\
\hline 2005 & $2.26 \%$ & $6.07 \%$ & 7.79\% & $18.25 \%$ & $0.36 \%$ & $1.51 \%$ & $1.01 \%$ & $2.73 \%$ \\
\hline 2006 & $3.43 \%$ & $0.30 \%$ & $8.12 \%$ & $11.12 \%$ & $1.04 \%$ & $6.48 \%$ & $0.60 \%$ & $1.99 \%$ \\
\hline 2007 & $11.64 \%$ & $0.65 \%$ & $9.49 \%$ & $8.38 \%$ & $0.92 \%$ & $7.34 \%$ & $0.10 \%$ & $7.32 \%$ \\
\hline 2008 & $5.89 \%$ & $0.58 \%$ & $15.16 \%$ & $14.80 \%$ & $2.25 \%$ & $2.22 \%$ & $0.21 \%$ & $0.52 \%$ \\
\hline 2009 & $7.74 \%$ & $1.61 \%$ & $7.70 \%$ & $14.55 \%$ & $1.46 \%$ & $1.97 \%$ & $0.68 \%$ & $1.48 \%$ \\
\hline 2010 & $5.34 \%$ & $1.87 \%$ & $5.24 \%$ & $11.98 \%$ & $2.65 \%$ & $6.07 \%$ & $0.79 \%$ & $2.20 \%$ \\
\hline 2011 & $3.06 \%$ & $10.12 \%$ & $5.35 \%$ & $12.55 \%$ & $6.07 \%$ & $1.17 \%$ & $2.52 \%$ & $1.71 \%$ \\
\hline Total & $6.99 \%$ & $1.62 \%$ & $7.43 \%$ & $9.00 \%$ & $1.40 \%$ & $2.35 \%$ & $1.98 \%$ & $10.59 \%$ \\
\hline
\end{tabular}


Table V: Geographic Distribution of Sites

\begin{tabular}{|c|c|c|c|c|}
\hline \multicolumn{3}{|c|}{ (a) US vs. Ex-US } & \multicolumn{2}{|c|}{ (b) Ex-US } \\
\hline Year & US & RoW & OECD & Other \\
\hline 1989 & $81.82 \%$ & $18.18 \%$ & $100.00 \%$ & $0.00 \%$ \\
\hline 1990 & $77.17 \%$ & $22.83 \%$ & $99.11 \%$ & $0.89 \%$ \\
\hline 1991 & $67.51 \%$ & $32.49 \%$ & $99.83 \%$ & $0.17 \%$ \\
\hline 1992 & $49.19 \%$ & $50.81 \%$ & $99.79 \%$ & $0.21 \%$ \\
\hline 1993 & $47.31 \%$ & $52.69 \%$ & $99.59 \%$ & $0.41 \%$ \\
\hline 1994 & $46.57 \%$ & $53.43 \%$ & $99.64 \%$ & $0.36 \%$ \\
\hline 1995 & $47.50 \%$ & $52.50 \%$ & $99.54 \%$ & $0.46 \%$ \\
\hline 1996 & $55.23 \%$ & $44.77 \%$ & $99.72 \%$ & $0.28 \%$ \\
\hline 1997 & $48.18 \%$ & $51.82 \%$ & $98.87 \%$ & $1.13 \%$ \\
\hline 1998 & $46.09 \%$ & $53.91 \%$ & $98.06 \%$ & $1.94 \%$ \\
\hline 1999 & $53.49 \%$ & $46.51 \%$ & $96.62 \%$ & $3.38 \%$ \\
\hline 2000 & $56.33 \%$ & $43.67 \%$ & $92.05 \%$ & $7.95 \%$ \\
\hline 2001 & $56.54 \%$ & $43.46 \%$ & $89.06 \%$ & $10.94 \%$ \\
\hline 2002 & $54.08 \%$ & $45.92 \%$ & $90.19 \%$ & $9.81 \%$ \\
\hline 2003 & $52.69 \%$ & $47.31 \%$ & $90.66 \%$ & $9.34 \%$ \\
\hline 2004 & $54.46 \%$ & $45.54 \%$ & $84.91 \%$ & $15.09 \%$ \\
\hline 2005 & $62.75 \%$ & $37.25 \%$ & $83.32 \%$ & $16.68 \%$ \\
\hline 2006 & $85.10 \%$ & $14.90 \%$ & $83.50 \%$ & $16.50 \%$ \\
\hline 2007 & $87.54 \%$ & $12.46 \%$ & $58.89 \%$ & $41.11 \%$ \\
\hline 2008 & $68.82 \%$ & $31.18 \%$ & $55.36 \%$ & $44.64 \%$ \\
\hline 2009 & $77.65 \%$ & $22.35 \%$ & $62.25 \%$ & $37.75 \%$ \\
\hline 2010 & $76.56 \%$ & $23.44 \%$ & $68.87 \%$ & $31.13 \%$ \\
\hline 2011 & $68.03 \%$ & $31.97 \%$ & $53.59 \%$ & $46.41 \%$ \\
\hline Total & $55.65 \%$ & $44.35 \%$ & $94.61 \%$ & $5.39 \%$ \\
\hline
\end{tabular}

Table entries are the fraction of investigator contracts in that year located in each geographic area. Panel (a) shows the breakdown between US and all other countries. Panel B breaks out the Ex-US countries into OECD member countries (Canada, Austria, Belgium, Ireland,

Luxembourg, Monaco, Netherlands, Switzerland, Finland, France, Norway, Germany, Italy, Spain, Sweden, Denmark, Japan, Australia, New Zealand, United Kingdom) versus all others. Based on 216,929 total observations. 
TABLE VI: PARAMETER ESTIMATES ON LOG PATIENTS AND SITE WORK EFFORT TRIAL CHARACTERISTICS VARIABLES

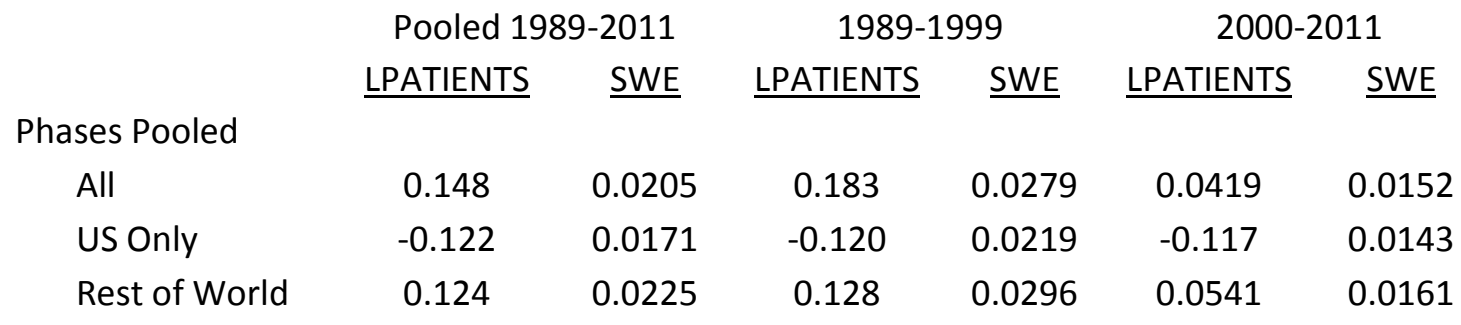

By Phase

All

Phase I

Phase II

$-0.145 \quad 0.0130$

$-0.168$

0.0164

$-0.0966$

0.00972

Phase IIIA

$-0.0173$

0.0182

$-0.011^{\dagger}$

0.0222

$-0.0321$

0.0149

Phase IIIB

0.120

0.0198

0.173

0.0285

$-0.0240$

0.0137

Phase IV

0.155

0.0284

0.226

0.0341

0.0299

0.0244

0.320

0.0344

0.305

0.0489

0.272

0.0253

By Phase

us Only

Phase I

Phase II

$$
-0.176
$$

0.0143

$-0.219$

0.0194

$-0.104$

0.0102

Phase IIIA

$-0.164$

0.0159

$-0.197$

0.0173

$-0.125$

0.0144

Phase IIIB

$-0.102$

0.0164

$-0.0986$

0.0239

$-0.0940$

0.0127

Phase IV

$-0.0631$

0.0257

$-0.0541$

$0.0238-0.0513$

0.0263

$-0.170$

0.0247

$-0.153$

0.0273

$-0.190$

0.0217

By Phase

Rest of World

Phase I

$-0.120$

0.0109

$-0.131$

0.0134

$-0.0799$

0.00788

Phase II

$-0.01$

0.0186

$-0.0313$

0.0265

$-0.0080^{\dagger}$

0.0143

Phase IIIA

0.0826

0.0220

0.121

0.0277

$-0.0588$

0.0157

Phase IIIB

0.101

0.0276

0.115

0.0372

0.0409

0.0213

Phase IV

0.217

0.0482

0.171

0.0619

0.225

0.0322

Notes: Table entries are the estimated coefficients on LPATIENTS and SWE in a regression of $\log$ (TGPP) on these and other explanatory variables. With the exception of the coefficients marked $\uparrow$, all coefficients in the table were statistically distinguishable from zero at the $p<0.01$ level using robust standard errors, clustered by trial protocol. The phases pooled regression includes a constant, indicator variables for therapeutic class, trial phase, and years. The by phase regressions include a constant and indicator variables for therapeutic class and year. Number of observations was 207,950 in the top panel All regressions, 118,477 in the US Only regressions, and 89,473 in the Rest of World regressions. 
TABLE VII: AVERAGE ANNUAL GROWTH RATES OF CLINICAL TRIAL COSTS ALTERNATIVE MODELS AND TIME PERIODS

\begin{tabular}{|c|c|c|c|c|c|}
\hline & \multicolumn{3}{|c|}{ 1989-2011 Regression AAGR } & \multirow{2}{*}{$\begin{array}{l}\text { 1989-1999 } \\
\text { Regression }\end{array}$} & \multirow{2}{*}{$\begin{array}{l}2000-2011 \\
\text { Regression }\end{array}$} \\
\hline & 1989- & 1989- & 1999- & & \\
\hline & 2011 & 1999 & 2011 & AAGR & AAGR \\
\hline \multicolumn{6}{|l|}{ Base Model^^} \\
\hline All & $6.96 \%$ & $3.98 \%$ & $9.45 \%$ & $3.79 \%$ & $9.93 \%$ \\
\hline US Only & 7.01 & 5.80 & 8.01 & 5.70 & 7.38 \\
\hline Rest of World & 8.70 & 6.66 & 10.39 & 6.54 & 12.36 \\
\hline
\end{tabular}

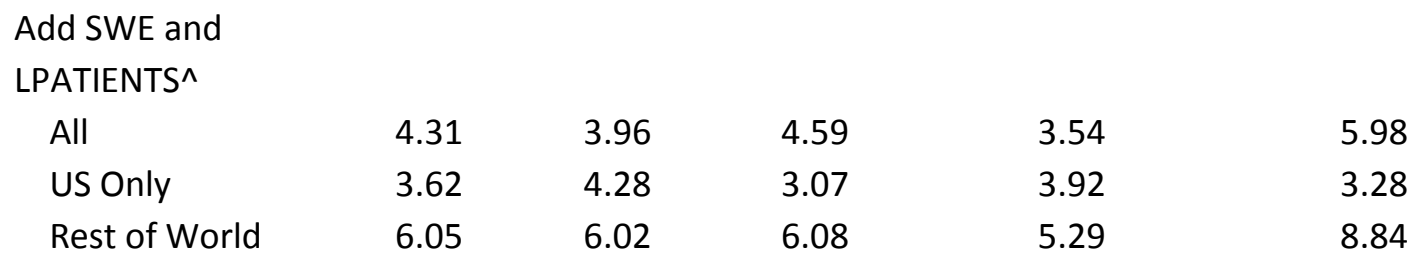

With SWE and LPATIENTS by phase $^{\wedge \wedge}$

All

$\begin{array}{lccccc}\text { Phase I } & 7.48 \% & 5.67 \% & 8.98 \% & 5.19 \% & 9.08 \% \\ \text { Phase II } & 6.01 & 6.58 & 5.54 & 6.91 & 5.98 \\ \text { Phase IIIA } & 4.04 & 3.88 & 4.18 & 3.25 & 5.16 \\ \text { Phase IIIB } & 3.78 & 2.32 & 5.00 & 1.79 & 5.65 \\ \text { Phase IV } & 11.91 & 9.25 & 14.13 & 8.17 & 15.75 \\ \text { US Only } & & & & & \\ \text { Phase I } & 6.78 & 5.72 & 7.66 & 5.07 & 8.58 \\ \text { Phase II } & 6.00 & 7.77 & 4.52 & 7.61 & 4.32 \\ \text { Phase IIIA } & 3.78 & 4.33 & 3.32 & 3.68 & 2.93 \\ \text { Phase IIIB } & 3.48 & 3.92 & 3.11 & 4.24 & 3.34 \\ \text { Phase IV } & 6.43 & 8.02 & 5.11 & 7.80 & 4.03 \\ \text { Rest of World } & & & & & \\ \text { Phase I } & 15.51 & 11.69 & 18.70 & 10.62 & 23.96 \\ \text { Phase II } & 7.64 & 8.48 & 6.95 & 8.65 & 9.52 \\ \text { Phase IIIA } & 6.29 & 6.87 & 5.81 & 6.30 & 7.87 \\ \text { Phase IIIB } & 12.29 & 15.54 & 9.59 & 13.72 & 11.55 \\ \text { Phase IV } & 9.36 & 10.73 & 8.22 & 8.87 & 11.74\end{array}$

Notes: Table entries are the annual average growth rate (AAGR) of an hedonic price index constructed from the estimated coefficients on indicator variables for year. See text. $\wedge^{\wedge}$ Regressions also include constant and indicator variables for therapeutic class, and trial phase. ${ }^{\wedge} \wedge$ Regressions also include constant and indicator variables for therapeutic class. 


\section{TABLE VIII: AVERAGE ANNUAL GROWTH RATES OF CLINICAL TRIAL COSTS BY THERAPEUTIC AREA AND TIME PERIOD}

$\underline{\text { Therapeutic Class }}$

Anti-infective

Cardiovascular

Central Nervous System

Dermatology

Devices \& Diagnostics

Endocrine

Gastrointestinal

Genitourinary System

Hematology

Immunomodulation

Oncology

Ophthalmology

Pain \& Anaesthesia

Pharmacokinetics

Respiratory System

Therapeutic Class

Anti-infective

Cardiovascular

Central Nervous System

Dermatology

Devices \& Diagnostics

Endocrine

Gastrointestinal

Genitourinary System

Hematology

Immunomodulation

Oncology

Ophthalmology

Pain \& Anaesthesia

Pharmacokinetics

Respiratory System

\author{
1989-2011 Regression \\ 1989-2011 AAGR
}

$4.73 \%$
18.64
5.05
6.56
$17.62^{\mathrm{a}}$
4.94
7.11
9.02
11.17
6.08
6.70
9.67
10.07
6.84
8.56


-2011 Regression

$4.73 \%$

18.64

5.05

6.56

7.11

6.08

6.70

1989-99 Regression 1989-99 AAGR

US Only Regressions

$0.81 \%$
4.95
5.00
9.18
$18.32^{\mathrm{b}}$
5.60
5.77
9.96
25.24
6.77
3.81
8.96
9.59
5.27
5.84

99 Regression
$89-99$ AAGR

$7.65 \%$

14.10

5.35

2.10

22.08

4.20

3.14

8.87

2.71

5.82

6.51

21.97

11.25

8.98

12.13 \begin{tabular}{cc}
$\begin{array}{c}\text { 1989-99 Regression } \\
\text { 1989-99 AAGR }\end{array}$ & 2000-2011 Regression \\
\hline
\end{tabular}

Rest of World Regressions

$\begin{array}{ccc}10.19 \% & 12.66 \% & 14.13 \% \\ 15.88 & 13.24 & 13.95 \\ 9.68 & 6.18 & 13.32 \\ 15.99^{\mathrm{c}} & 15.06 & -1.41 \\ 17.50^{\mathrm{d}} & -9.97^{\mathrm{g}} & 30.61^{\mathrm{h}} \\ 6.10 & 7.66 & 5.83 \\ 20.14 & 3.27 & 23.55 \\ 6.58 & 4.75 & 9.01 \\ 11.40^{\mathrm{e}} & 19.24 & 2.38^{\mathrm{i}} \\ 13.17 & 18.07 & 12.01 \\ 9.60 & 6.39 & 14.40 \\ 29.60 & 42.85 & 17.69 \\ 43.98^{\mathrm{f}} & 47.61 & 45.37 \\ 7.99^{\mathrm{f}} & 1.65 & 12.93^{\mathrm{k}} \\ 11.52 & 10.25 & 20.09^{\mathrm{k}}\end{array}$

Notes: ${ }^{\mathrm{a}} 1999-2011 ;{ }^{\mathrm{b}} 1990-94 ;{ }^{\mathrm{c}} 1990-2003 ;{ }^{\mathrm{d}} 1992-98 ;{ }^{\mathrm{e}} 1989-2005 ;{ }^{\mathrm{f}} 1989-2006 ;{ }^{\mathrm{g}} 1993-99 ;{ }^{\mathrm{h}} 2009-11$; ${ }^{\mathrm{i}} 2000-5 ;{ }^{\mathrm{k}} 2000-06$ 


\title{
TABLE IX: PARAMETER ESTIMATES IN MODEL ESTIMATED AT THE TRIAL LEVEL WITH ADDITIONAL TRIAL-SPECIFIC EXPLANATORY VARIABLES, POOLED 1989-2011
}

\author{
DEPENDENT VARIABLE: In TGPP
}

No. Sites

No. Sites/Country

Sample Size

Pooled Over Phases

$0.00164^{* * *}$

$-0.000979$

24,172

By Phase

Phase I

0.0223

0.0102

5,557

Phase II

0.00116

$0.00506 * * *$

5,775

Phase IIIA

0.000371

0.00161 *

8,953

Phase IIIB

$-0.000837$

0.000454

1,735

Phase IV

$-0.00206$

$-0.00126$

2,152

Notes: $* * *, * *$ and $*$ denote statistical significance at p-values of $0.01,0.05$ and 0.10 , respectively. The phases pooled regression includes a constant, LPATIENTS, SWE, and indicator variables for therapeutic class, trial phase and years. The by phase regressions include a constant, LPATIENTS, SWE, and indicator variables for therapeutic class and year. Regressions are pooled into All, 1989-2011. 


\section{Figure 1: Site Work Effort (SWE) Over Time}

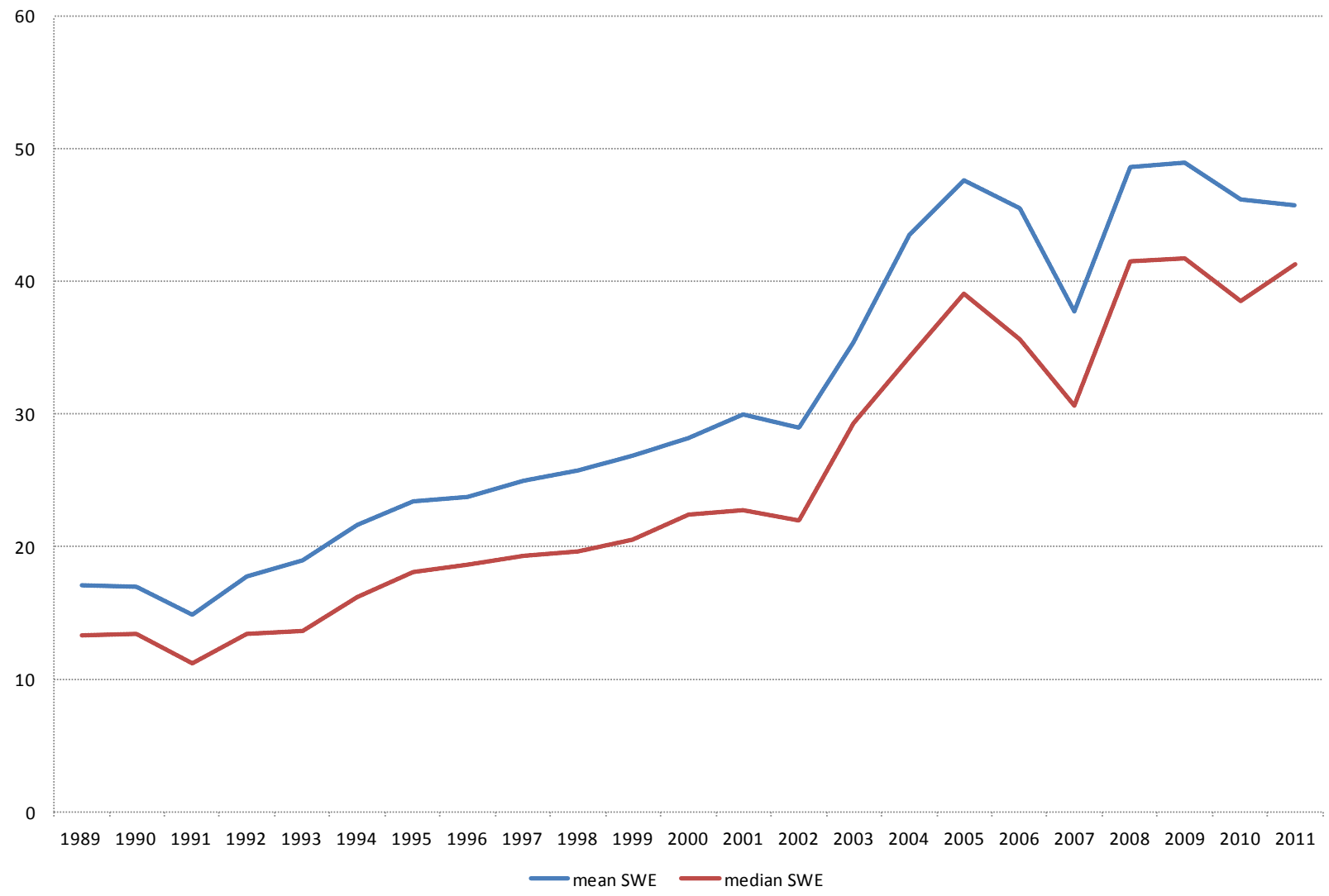

Figure 2: Patients per Site Over Time

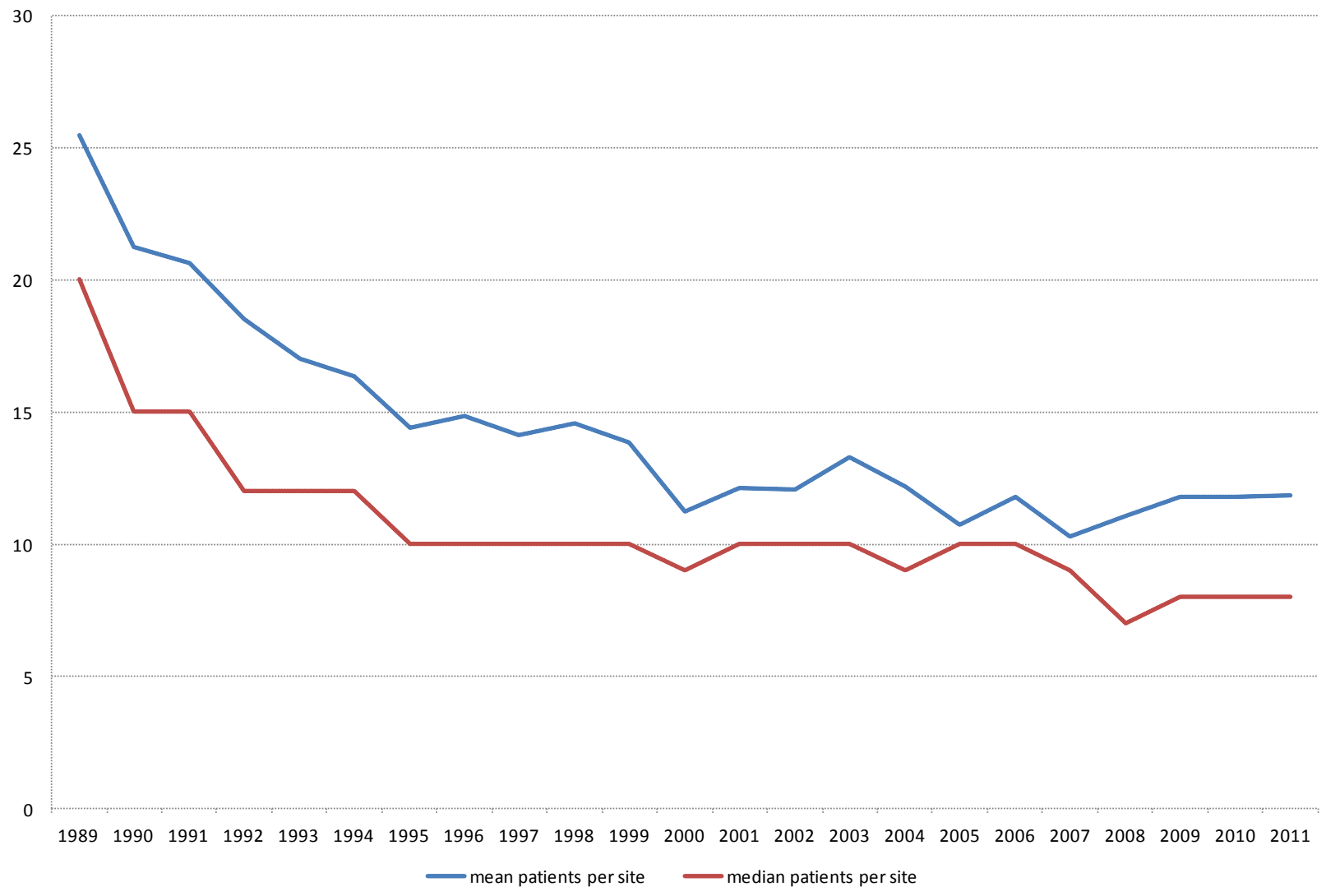


Figure 3: Nominal TGPP, BRDPI, and Hedonic Indexes

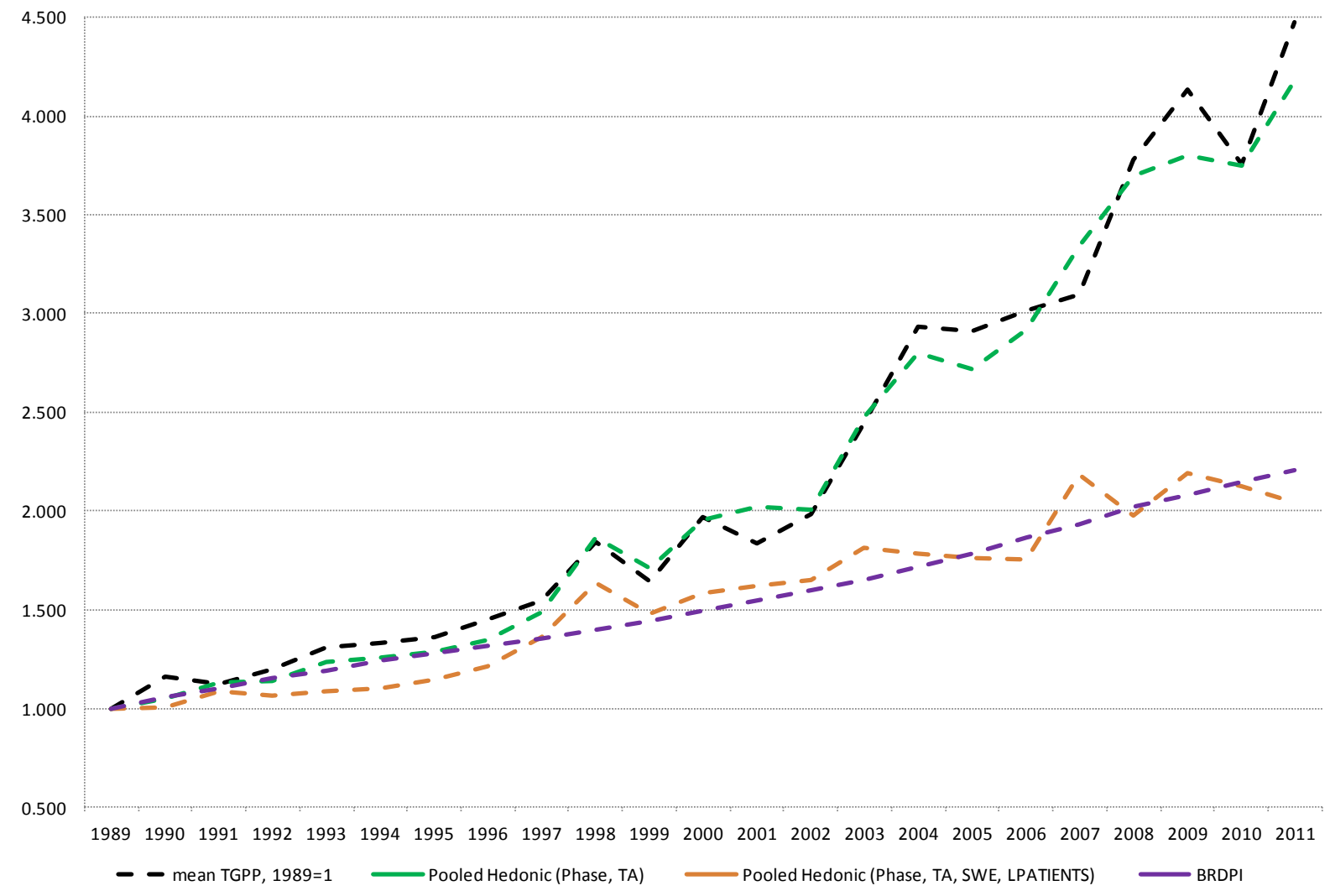

Figure 4: Alternate Hedonic Indexes

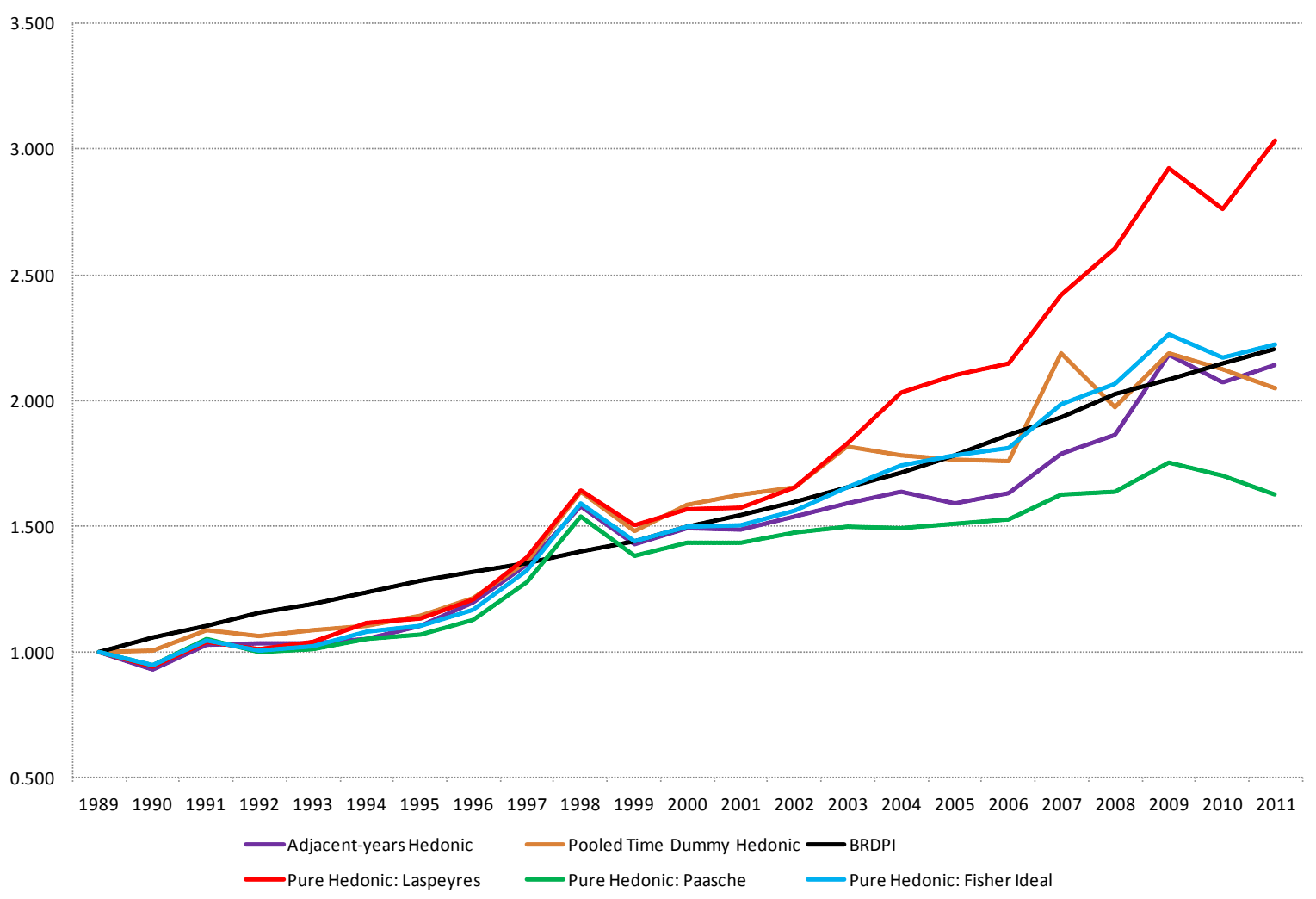

